\title{
GLOBAL ANALYSIS OF TWO-PARAMETER ELLIPTIC EIGENVALUE PROBLEMS
}

\author{
BY \\ H. O. PEITGEN ${ }^{1}$ AND K. SCHMITT ${ }^{2}$
}

\begin{abstract}
We consider the nonlinear boundary value problem (*) $L u+\lambda f(u)=0$, $x \in \Omega, u=\sigma \phi, x \in \partial \Omega$, where $L$ is a second order elliptic operator and $\lambda$ and $\sigma$ are parameters. We analyze global properties of solution continua of these problems as $\lambda$ and $\sigma$ vary. This is done by investigating particular sections, and special interest is devoted to questions of how solutions of the $\sigma=0$ problem are embedded in the two-parameter family of solutions of $(*)$. As a natural biproduct of these results we obtain (a) a new abstract method to analyze bifurcation from infinity, (b) an unfolding of the bifurcations from zero and from infinity, and (c) a new framework for the numerical computations, via numerical continuation techniques, of solutions by computing particular one-dimensional sections.
\end{abstract}

1. Introduction. In some earlier work [9-11] we considered the nonlinear oneparameter elliptic boundary value problem

$$
\begin{aligned}
& L u+\lambda f(u)=0, \quad x \in \Omega, \\
& u=0, \quad x \in \partial \Omega,
\end{aligned}
$$

where $L$ is a second-order partial differential operator which is uniformly elliptic in the bounded domain $\Omega \subset \mathbf{R}^{n}$ with smooth boundary $\partial \Omega$, and $f$ is a nonlinearity which is linear at infinity, i.e.

$$
f(s) / s \rightarrow m_{\infty} \text { as }|s| \rightarrow \infty,
$$

and $f(0)=0$, and $f^{\prime}(0)=m_{0}>0$. We demonstrated, via topological perturbation techniques, that this problem has a global branch of positive solutions and a global branch of negative solutions which bifurcate from infinity at $\lambda_{0}^{\infty}=\alpha_{0} m_{\infty}^{-1}$, where $\alpha_{0}$ is the principal eigenvalue of the linear problem

$$
\begin{aligned}
L u+\alpha u & =0, & & x \in \Omega, \\
u & =0, & & x \in \partial \Omega
\end{aligned}
$$

(see also [2]). Our method of proof was such that the perturbed problem (artificially introduced) had the property that these branches bifurcating from infinity could, in fact, be obtained from branches which bifurcate from the trivial solution, thus obviating a bifurcation analysis in a neighborhood of infinity. This approach, in fact, permitted us to treat a broader class of nonlinearities which were not necessarily

Received by the editors February 3, 1983.

1980 Mathematics Subject Classification. Primary 35J60, 35J65, 34B15; Secondary 35B30, 35B45, $47 \mathrm{H} 15$.

${ }^{1}$ Supported by Stiftung Volkswagenwerk.

2 Supported by NSF Grant MCS8121951. 
linear at infinity. A further advantage of this approach was exploited in [10 and 12], where we discussed finite-dimensional approximations of (1.1) and used numerical continuation methods to compute such branches bifurcating from infinity.

In the present paper we continue the study of this class of problems by embedding (1.1) into a natural two-parameter problem, $(\lambda, \sigma) \in \mathbf{R} \times \mathbf{R}$,

$$
\begin{aligned}
L u+\lambda f(u) & =0, & & x \in \Omega, \\
u & =\sigma \phi, & & x \in \partial \Omega,
\end{aligned}
$$

where $\phi \not \equiv 0$ are given boundary data. Naturally, one expects that the set of solutions for (1.3) is given by two-dimensional continua. Our main interest in this paper is to analyze their global properties as $\lambda$ and $\sigma$ vary. This is done by investigating particular one-dimensional sections, and special interest is devoted to the question how solutions of (1.1) are embedded in the two-parameter families of solutions of (1.3). In this regard we shall obtain, as a biproduct,

a new abstract method to analyze bifurcation from infinity (see also [14]);

bifurcation results from infinity for (1.1) at higher eigenvalues of (1.2);

an unfolding of the bifurcations from zero and infinity for (1.1);

a new frame for the numerical computation, via numerical continuation

techniques, of solutions of (1.1) by computing particular one-dimensional

sections of (1.3).

Our method is a blend of linearization and continuation techniques. A key role is played by the fact that, for fixed $\lambda$ and $|\sigma| \gg 1$, problem (1.3) is essentially equivalent to a linear problem which is uniquely solvable (see (3.4)).

In $\$ 2$ we collect preliminary results and notation. $\$ 3$ is devoted to the general theory of (1.3), and in $\$ 4$ we study particular one-dimensional sections of (1.3). $\S 5$ restricts to a particular ODE-model of (1.3), for which we will obtain a much more complete picture of the solution set and encounter new phenomena like secondary bifurcation. Finally, in $\$ 6$ we present some numerical studies and experiments. These were carried out by D. Saupe (Universität Bremen) in the Compuer Aided Geometric Design Laboratory of the University of Utah and were supported by a grant from 'Stiftung Volkswagenwerk'.

One of the main objectives of the paper is to analyze many subcontinua of solutions of (1.3). This requires a great amount of technical notation. In order that it be understood more easily we recommend that Remark 5.1 be read together with the figures of $\S 2$.

2. Notation and preliminary results. Let $\Omega$ be a bounded domain in $\mathbf{R}^{n}$ with smooth boundary $\partial \Omega$ (i.e. $\partial \Omega$ is of class $C^{2+\alpha}$ for some $\alpha \in(0,1)$ ). Let $a_{i j}, b_{i}, 1 \leqslant i, j \leqslant n$, be real valued and Lipschitz continuous on $\operatorname{cl} \Omega$ and let the matrix $\left(a_{i j}(x)\right)$ be positive definite on $\mathrm{cl} \Omega$. Let $L$ denote the uniformly elliptic operator

$$
L u=\sum_{i, j=1}^{n} a_{i j}(x) \frac{\partial^{2} u}{\partial x_{i} \partial x_{j}}+\sum_{j=1}^{n} b_{j}(x) \frac{\partial u}{\partial x_{j}}
$$


and assume that $L$ is selfadjoint in $H_{0}^{1}(\Omega)$. Let $\phi: \operatorname{cl} \Omega \rightarrow \mathbf{R}$ and $f: \mathbf{R} \rightarrow \mathbf{R}$ be Lipschitz continuous satisfying the following conditions:

$$
\begin{aligned}
& f(s)=m_{0} s+o(s) \quad \text { as } s \rightarrow 0, \\
& f(s)=m_{\infty} s+o(s) \quad \text { as }|s| \rightarrow \infty, \\
& m_{0}>0, \quad m_{\infty}>0 .
\end{aligned}
$$

We consider the nonlinear Dirichlet problem

$$
\begin{aligned}
L u+\lambda f(u) & =0, & & x \in \Omega, \\
u & =\sigma \phi, & & x \in \partial \Omega,
\end{aligned}
$$

where $\lambda$ and $\sigma$ are real parameters. Let $\alpha_{0}<\alpha_{1} \leqslant \alpha_{2} \leqslant \alpha_{2} \leqslant \cdots$ denote the eigenvalues of the linear problem

$$
\begin{aligned}
L u+\alpha u=0, & x \in \Omega, \\
u=0, & x \in \partial \Omega,
\end{aligned}
$$

and let

$$
\lambda_{i}^{0}=\alpha_{i} m_{0}^{-1}, \quad \lambda_{i}^{\infty}=\alpha_{i} m_{\infty}^{-1}, \quad i=0,1,2, \ldots
$$

It follows from the maximum principle, the Fredholm alternative and regularity theory (see [4]), that the problem

$$
\begin{aligned}
L u=0, & x \in \Omega, \\
u=\phi, & x \in \partial \Omega,
\end{aligned}
$$

has a unique solution, say $w$, and that (2.3) is equivalent to the operator equation

$$
u=\lambda \varrho F(u)+\sigma w,
$$

where $\mathcal{L}: E \rightarrow E, E=C^{0}(\mathrm{cl} \Omega)$, is a compact linear operator and $F: E \rightarrow E$ is the Nemits'kii operator induced by $f$, which is a continuous operator.

We shall be interested in ( $\lambda, \sigma)$-families of solutions $(\lambda, \sigma, u)$ of (2.6) in $\mathbf{R} \times \mathbf{R} \times$ $E$. We find it convenient to distinguish the $\lambda$ - and $\sigma$-parameter spaces by denoting

$$
\lambda \in \mathbf{R}=\Lambda \text { and } \boldsymbol{\sigma} \in \mathbf{R}=\Sigma .
$$

Thus, speaking of a $\sigma$-continuum (respectively $\lambda$-continuum) we mean a closed and connected subset of solutions of (2.6) in $\Sigma \times E$ for $\lambda$ fixed (respectively in $\Lambda \times E$ for $\sigma$ fixed).

To establish the existence of solutions we will make extensive use of two basic results in the context of topological continuation:

LEMMA 2.1 (SEE [6, 21]). Let $K$ be a compact metric space and $A, B$ closed, nonempty subsets. Then either $A, B$ are separated in $K$, i.e.

$$
K=K_{A} \cup K_{B}, \quad A \subset K_{A}, \quad B \subset K_{B} \text { and } K_{A} \cap K_{B}=\varnothing, \quad K_{A} \text { and } K_{B} \text { closed; }
$$

or $A$ and $B$ are connected in $K$, i.e., there exists a continuum $\circlearrowright \subset K$ such that

$$
\bigodot \cap A \neq \varnothing \text { and } \circlearrowright \cap B \neq \varnothing \text {. }
$$

THEOREM 2.1 (SEE [17]). Let $E$ be a Banach space and $\Gamma \subset E \times[a, b]$ an open and bounded subset. Let $h$ : $\mathrm{cl} \Gamma \rightarrow E$ be completely continuous and assume that $0 \notin$ $(\mathrm{id}-h)(\partial \Gamma)$. Then

$$
\operatorname{deg}\left(\mathrm{id}-h(\cdot, \lambda), \Gamma_{\lambda}, 0\right)=\text { constant }
$$


Here deg denotes the Leray-Schauder degree and

$$
\Gamma_{\lambda}=\{u \in E:(u, \lambda) \in \Gamma\}
$$

is the $\lambda$-section of $\Gamma$ in $E \times \mathbf{R}$. The Leray-Schauder continuation principle asserts that there exists a continuum $C \subset(\text { id }-h)^{-1}(0)$ with

$$
e_{a} \neq \varnothing \neq e_{b}
$$

provided $\operatorname{deg}\left(\mathrm{id}-h(\cdot, a), \Gamma_{a}, 0\right) \neq 0$, and $0 \notin(\mathrm{id}-h)(\partial \Gamma)$. This is a consequence of Lemma 2.1 and Theorem 3.1. We also, however, need a generalization of this principle to an infinite interval of parameters.

THEOREM 2.2 (SEE [19]). Let $E$ be a Banach space and let $\Gamma \subset E \times(a, b)$ be an open subset $(a=-\infty, b=\infty$ not excluded $)$ such that, for each compact subinterval $\left[a_{0}, b_{0}\right] \subset(a, b), \Gamma \cap E \times\left[a_{0}, b_{0}\right]$ is bounded. Let $h: \mathrm{cl} \Gamma \rightarrow E$ be completely continuous and assume that $0 \notin(\mathrm{id}-h)(\partial \Gamma)$. Furthermore, assume that

$$
\operatorname{deg}\left(\mathrm{id}-h(\cdot, \lambda), \Gamma_{\lambda}, 0\right) \neq 0
$$

for some $\lambda \in(a, b)$. Then there exists a continuum $\subset \subset(\mathrm{id}-h)^{-1}(0)$ such that

$$
\pi(\mathcal{C})=(a, b)
$$

where $\pi$ denotes the projection $\pi: E \times \mathbf{R} \rightarrow \mathbf{R},(u, \lambda) \rightarrow \lambda$.

We also need a slight modification of this result, which we prove for reasons of completeness.

THEOREM 2.3. Let $E$ be a Banach space and let $h: E \times[a, b] \rightarrow E$ be completely continuous. Assume that there is an $R>0$ such that $h(u, a) \neq u \neq h(u, b)$ for all $u$ with $\|u\| \geqslant R$. Furthermore, assume that

$$
\begin{gathered}
\operatorname{deg}(\mathrm{id}-h(\cdot, a), B(0, R), 0) \neq \operatorname{deg}(\mathrm{id}-h(\cdot, b), B(0, R), 0) . \\
(B(0, R)=\{u:\|u\|<R\} .)
\end{gathered}
$$

Then there exists a continuum $C \subset(\mathrm{id}-h)^{-1}(0)$ which is unbounded in $E \times[a, b]$.

Proof. Let $S_{r}=\{u \in E:\|u\|=r\}$. Then

$$
A(r)=\left(S_{r} \times[a, b]\right) \cap(\mathrm{id}-h)^{-1}(0) \neq \varnothing
$$

for any $r \geqslant R$ as a consequence of the homotopy property of the Leray-Schauder degree. We prove the assertion in two steps.

Step 1 . For any $r>R$ there exists a continuum $\Theta_{r} \subset(\mathrm{id}-h)^{-1}(0)$ which connects $A(R)$ with $A(r)$. If not, let

$$
K=(\mathrm{id}-h)^{-1}(0) \cap \mathrm{cl}(B(0, r) \backslash B(0, R)) \times[a, b] .
$$

Then $K$ is compact and $A(R), A(r)$ are disjoint, closed and nonernpty subsets. Now, by Lemma 2.1 we may find an open and bounded subset

$$
\Gamma \subset(B(0, r) \backslash B(0, R)) \times[a, b]=X
$$

such that

$$
\partial \Gamma \cap(\mathrm{id}-h)^{-1}(0)=\varnothing \quad(\partial \text { in } X),
$$


and $A(R) \subset \Gamma$. Define $\theta:=B(0, R) \times[a, b] \cup \Gamma$. Then

$$
\partial \vartheta \cap(\mathrm{id}-h)^{-1}(0)=\varnothing \quad(\partial \text { in } E \times[a, b]),
$$

which contradicts the generalized homotopy property, Theorem 2.1 .

Step 2. Let $(u, \lambda) \in A(R)$ and let $\delta(u, \lambda)$ be the maximal component in (id $-h)^{-1}(0)$, which contains $(u, \lambda)$. We have to show that there is a $(u, \lambda) \in A(R)$ such that $\delta(u, \lambda)$ is unbounded. Assume that this is not the case. Thus, for each $(u, \lambda) \in A(R)$ there exists an $r=r(u, \lambda)$ such that

$$
\delta(u, \lambda) \subset B(0, r) \times[a, b] .
$$

Defining $K$ as in Step 1 we may apply Lemma 2.1 again to conclude that $\delta(u, \lambda)$ and $A(r)$ are separated in $K$. Thus, we may find an open and bounded subset

$$
\Gamma(u, \lambda) \subset(B(0, r) \backslash B(0, R)) \times[a, b]=X
$$

such that

$$
\partial \Gamma(u, \lambda) \cap(\mathrm{id}-h)^{-1}(0)=\varnothing \quad(\partial \text { in } X)
$$

and $\delta(u, \lambda) \subset \Gamma(u, \lambda)$. Observe that

$$
\Gamma(u, \lambda) \cap S_{R} \times[a, b]=W(u, \lambda)
$$

is an open neighborhood of $(u, \lambda)$ in $S_{R} \times[a, b]=Y$ with no solutions on $\partial W(u, \lambda)$ ( $\partial$ in $Y$ ). Since $A(R)$ is compact, we may find

$$
\left(u_{1}, \lambda_{1}\right), \ldots,\left(u_{k}, \lambda_{k}\right) \in A(R) \text { and } r_{*}>R
$$

such that

$$
\bigcup_{i=1}^{k} W\left(u_{i}, \lambda_{i}\right) \supset A(R) \text { and } \bigcup_{i=1}^{k} \Gamma\left(u_{i}, \lambda_{i}\right) \subset B\left(0, r_{*}\right) \times[a, b] \text {. }
$$

Now, $A\left(r_{*}\right) \neq \varnothing$ and there exists $\bigodot_{r_{*}}$ connecting $A\left(r_{*}\right)$ with some element $\left(u_{*}, \lambda_{*}\right)$ $\in A(R)$ as a consequence of Step 1. However, $\left(u_{*}, \lambda_{*}\right)-W\left(u_{i}, \lambda_{i}\right)$, for some $i$, and hence $\partial \Gamma\left(u_{i}, \lambda_{i}\right) \cap \bigodot_{r_{*}} \neq \varnothing$. This is a contradiction.

Our analysis of (2.6) will also make extensive use of the asymptotic behavior of solutions of (2.6) in $\Sigma \times E$ for $\lambda$ fixed and $|\sigma| \gg 1$. We will see that the following problem can be considered to be a linearization at $|\sigma|=\infty$ :

$$
\begin{aligned}
L u+\lambda m_{\infty} u & =0, & & x \in \Omega, \\
u & =\phi, & & x \in \partial \Omega .
\end{aligned}
$$

Another essential ingredient for our problem (2.6) is that we suppose that the nonlinearity $f$ has a nontrivial zero-structure:

$$
\begin{aligned}
& s_{+}^{\infty}=\sup \{s: f(s)=0\}, \\
& s_{-}^{\infty}=\inf \{s: f(s)=0\}, \\
& s_{+}^{0}=\inf \{s>0: f(s)=0\}, \\
& s_{-}^{0}=\sup \{s<0: f(s)=0\}, \\
&-\infty<s_{-}^{\infty}<s_{-}^{0}<0<s_{+}^{0}<s_{+}^{\infty}<+\infty .
\end{aligned}
$$


In $[9,11]$ we studied problem (2.6) for positive (negative) solutions in $\Lambda \times E$ for $\sigma=0$ by means of a global perturbation $g(\lambda, s)$ of $\lambda f(s)$. Exploiting that perturbation we proved existence of continua of positive (negative) solutions bifurcating from infinity in $\Lambda \times E$ at $\lambda_{0}^{\infty}=\alpha_{0} m_{\infty}^{-1}$. Another consequence of the considerations there and in Theorem 2.1 is the following

LEMMA 2.2 (SEE [10, 11]). Assume that (2.8) is satisfied.

(a) Let $\lambda_{*}>\lambda_{0}^{0}$ be fixed. Then the following sets of solutions of (2.6) for $\sigma=0$ are nonempty:

$$
\begin{aligned}
\mathscr{P}_{0}\left(\lambda_{*}\right) & =\left\{\left(\lambda_{*}, 0, u\right) \text { solves }(2.6): u(x)>0 \text { for } x \in \Omega \text { and }\|u\|<s_{+}^{0}\right\}, \\
\Re_{0}\left(\lambda_{*}\right) & =\left\{\left(\lambda_{*}, 0, u\right) \text { solves }(2.6): u(x)<0 \text { for } x \in \Omega \text { and }\|u\|<-s_{-}^{0}\right\} .
\end{aligned}
$$

Furthermore, $\mathscr{P}_{0}\left(\lambda_{*}\right)$ and $\mathscr{\Re}_{0}\left(\lambda_{*}\right)$ are isolated in the set of all solutions in $E$ and if $U(\cdot)$ is an isolating neighborhood, then

$$
\operatorname{deg}\left(\mathrm{id}-\lambda_{*} \varrho F, U(\cdot), 0\right)=+1 .
$$

(b) Let $\lambda_{*}>\lambda_{0}^{\infty}$ be fixed. Then the following sets of solutions of (2.6) for $\sigma=0$ are nonempty:

$$
\begin{aligned}
\mathscr{P}_{\infty}\left(\lambda_{*}\right) & =\left\{\left(\lambda_{*}, 0, u\right) \text { solves }(2.6): u(x)>0 \text { for } x \in \Omega \text { and }\|u\|>s_{+}^{\infty}\right\}, \\
\mathcal{X}_{\infty}\left(\lambda_{*}\right) & =\left\{\left(\lambda_{*}, 0, u\right) \text { solves }(2.6): u(x)<0 \text { for } x \in \Omega \text { and }\|u\|>-s_{-}^{\infty}\right\} .
\end{aligned}
$$

Furthermore, $\mathscr{P}_{\infty}\left(\lambda_{*}\right)$ and $\mathcal{R}_{\infty}\left(\lambda_{*}\right)$ are isolated in the set of all solutions in $E$ and if $U(\cdot)$ is an isolating neighborhood, then

$$
\operatorname{deg}\left(\mathrm{id}-\lambda_{*} \varrho F, U(\cdot), 0\right)=-1 .
$$

The fact that the above sets are isolated follows from the strong maximum principle [4, p. 33]. Here $B(v, \delta)=\{u \in E:\|v-u\|<\delta\}$.

To provide some guideline for our abstract results in $\S \S 3-5$ we present 4 figures of $\sigma$-continua in $\Sigma \times E$ for the ODE-model

$$
\Omega=(0, \pi), \quad L=\frac{d^{2}}{d x^{2}}, \quad m_{0}=m_{\infty},
$$

and $\lambda=\lambda_{i}$ fixed with

$$
\begin{array}{cl}
0<\lambda_{0}<\lambda_{0}^{0}=\lambda_{0}^{\infty} & \text { (Figure 1), } \\
\lambda_{0}^{0}=\lambda_{0}^{\infty}<\lambda_{1}<\lambda_{1}^{0}=\lambda_{1}^{\infty} & \text { (Figure 2), } \\
\lambda_{1}^{0}=\lambda_{1}^{\infty}<\lambda_{2}<\lambda_{2}^{0}=\lambda_{2}^{\infty} & \text { (Figure 3), } \\
\lambda_{2}^{0}=\lambda_{2}^{\infty}<\lambda_{3}<\lambda_{3}^{0}=\lambda_{3}^{\infty} & \text { (Figure 4) }
\end{array}
$$

(further assumptions on $f$ are discussed in §5). All solutions of the ODE-model for (2.6) for $\lambda>0$ and $\sigma=0$ are characterized by a nodal structure, i.e. the number of internal zeros in $(0, \pi)$. We use the following notation:

$$
\begin{aligned}
& u_{i}^{0}\left(\lambda_{*}\right)=\quad \text { solution of the ODE-model for }(2.6), \lambda=\lambda_{*}, \sigma=0, \text { with } i \\
& \text { internal zeros in }(0,1), i=0,1, \ldots, \text { and }\left\|u_{i}^{0}\left(\lambda_{*}\right)\right\|<s^{0} . \\
& u_{i}^{\infty}\left(\lambda_{*}\right)=\quad \cdots \text { and }\left\|u_{i}^{\infty}\left(\lambda_{*}\right)\right\|>s^{\infty} .
\end{aligned}
$$

Here $s^{0}=\max \left\{s_{+}^{0}, \mid s_{-}^{0}\right\}, s^{\infty}=\min \left\{s_{+}^{\infty},\left|s_{-}^{\infty}\right|\right\}$. 

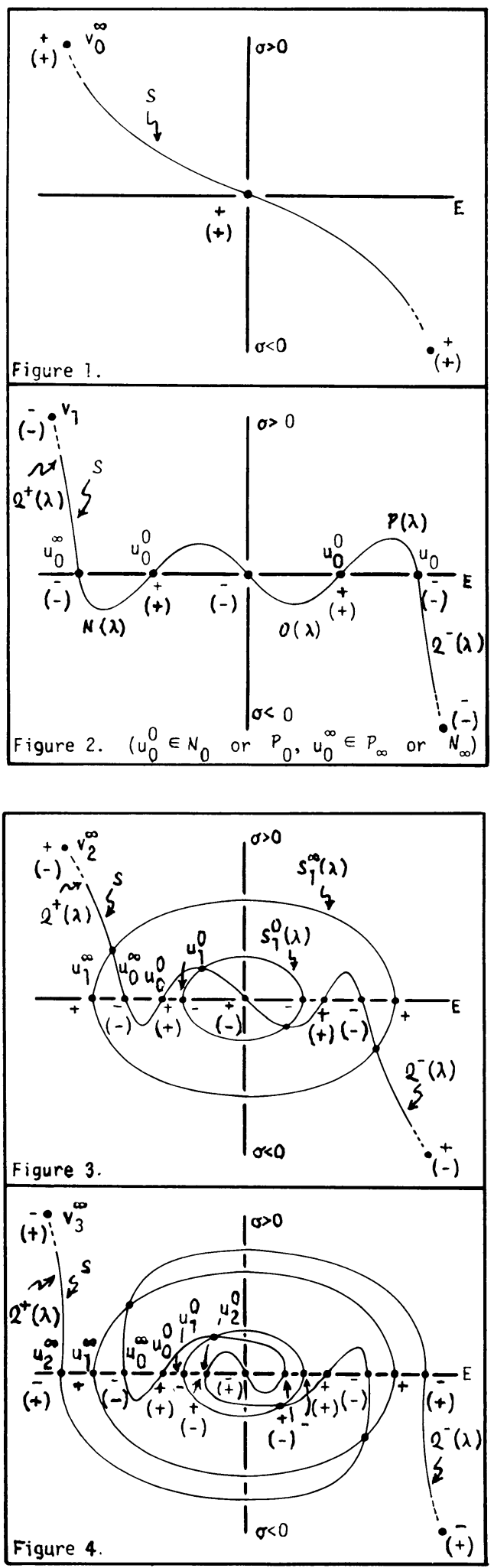
By $v_{k}^{\infty}(\lambda)$ we denote the unique solution (Fredholm alternative) of (2.7) for

$$
\lambda_{k-1}^{\infty}<\lambda<\lambda_{k}^{\infty}, \quad k=0,1, \ldots
$$

(where $\lambda_{-1}^{\infty}=\lambda_{-1}^{0}=0$ ). If $\phi(x) \equiv 1$, we give an explicit form for the solutions $v_{k}^{\infty}(\lambda)$ of

$$
\begin{gathered}
v^{\prime \prime}+\lambda m_{\infty} v=0, \quad v(0)=1=v(\pi), \\
v_{k}^{\infty}(\lambda)(x)=\frac{1-\cos \left(\sqrt{\lambda m_{\infty}} \pi\right)}{\sin \left(\sqrt{\lambda m_{\infty}} \pi\right)} \sin \left(\sqrt{\lambda m_{\infty}} x\right)+\cos \left(\sqrt{\lambda m_{\infty}} x\right) .
\end{gathered}
$$

In Figure 5 we give the graphs of $v_{0}^{\infty}, v_{1}^{\infty}, v_{2}^{\infty}$ and $v_{3}^{\infty}$.

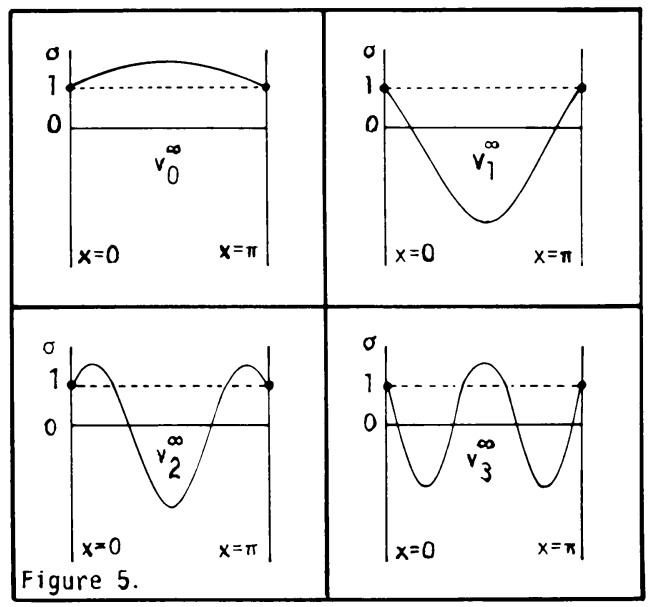

Note that the $v_{k}^{\infty}$ have a nodal-structure, which jumps by two as $k$ increases. This peculiar jump will play the key role in understanding Figures $1-4$. We will show in $\$ 5$ that Figures 1-4 represent $\sigma$-continua of solutions in $\Sigma \times E$ of the ODE-model for (2.6). In other words, we may regard Figures $1-4$ as representative $\lambda$-sections of a two-dimensional surface of solutions of (2.6) in $\Lambda \times \Sigma \times E$. With these figures in mind we may develop insight for the singularities in the $(\lambda, \sigma)$-problem.

Finally, we note an important property of solutions of the ODE-model,

$$
u^{\prime \prime}+\lambda f(u)=0, \quad u(0)=\sigma=u(\pi) .
$$

Let $\Phi^{x}$ denote the phase flow corresponding to the vectorfield

$$
V(u, v)=(v,-\lambda f(u))
$$

and let $S(u, v)=(u,-v)$. Note that $S^{2}=$ id and $S V S=-V$. Hence,

$$
\left(\Phi^{\times} S\right)^{2}=\left(S \Phi^{\times}\right)^{2}=\text { id for all } x \in \mathbf{R} \text {. }
$$

Thus, flow lines in the $\left(u, u^{\prime}\right)$-plane are symmetric with respect to a reflection in the $u$-axis and this implies that all solutions $u$ of $(2.13)$ for which $u^{\prime}(0) \neq u^{\prime}(\pi)$ are 
symmetric with respect to $\pi / 2$, i.e.

$$
u(\pi / 2-x)=u(\pi / 2+x) .
$$

This is true, in particular, for the solutions $v_{k}$.

3. General theory. Our goal in this section is to exploit the linear structure of (2.6) for $|\sigma| \gg 1$. We assume throughout this section that (2.1) and (2.2) are satisfied.

Lemma 3.1 ( $\sigma$-Continua). Let $\lambda \neq \lambda_{i}^{\infty}$ be fixed. Then for any $\rho>0$ there exists $R=R(\rho)>0$ such that if $|\sigma| \leqslant \rho$ and $(\lambda, \sigma, u)$ is a solution of $(2.6)$, then $\|u\| \leqslant R$.

Proof. Let $\rho>0$ be given and assume that the conclusion is false. Then there exists a sequence of solutions $\left\{\left(\lambda, \sigma_{n}, u_{n}\right)\right\},\left|\sigma_{n}\right| \leqslant \rho$ and $\left\|u_{n}\right\| \rightarrow \infty$ and

$$
u_{n}=\lambda \mathcal{E} F\left(u_{n}\right)+\sigma_{n} w .
$$

Dividing (3.1) by $\left\|u_{n}\right\|$, letting $v_{n}=u_{n}\left\|u_{n}\right\|^{-1}$ and recalling (2.2) we obtain

$$
v_{n}=\lambda m_{\infty} \varrho v_{n}+\lambda \varrho o\left(\left\|u_{n}\right\|\right)\left\|u_{n}\right\|^{-1}+\sigma_{n}\left\|u_{n}\right\|^{-1} w .
$$

Hence, $\left\{v_{n}\right\}$ has a subsequence converging to $v \in E,\|v\|=1$ and $v=\lambda m_{\infty} \ell v$, or equivalently, $v$ solves equation (2.4) for $\alpha=\lambda m_{\infty}$, i.e. $\alpha=\alpha_{i}$, for some $i$, contradicting that $\lambda \neq \lambda_{i}^{\infty}$, for any $i$.

From the above proof, in particular (2.16), we may deduce the following corollary.

Corollary 3.1 ( $\sigma$-CONTINUA). Let $\lambda \neq \lambda_{i}^{\infty}$ be fixed and let $\left\{\left(\lambda, \sigma_{n}, u_{n}\right)\right\}$ be $a$ sequence of solutions of (2.6) with

(a) $\left\|u_{n}\right\| \rightarrow \infty$, then $\left\{\sigma_{n}\left\|u_{n}\right\|^{-1}\right\}$ is a bounded sequence, which cannot have 0 as a limit point;

(b) $\left|\sigma_{n}\right| \rightarrow \infty$, then $\left\|u_{n}\right\| \rightarrow \infty$.

Proof. That $\left\{\sigma_{n}\left\|u_{n}\right\|^{-1}\right\}$ is a bounded sequence follows immediately from (3.2) and if 0 were a limit point of this sequence, we obtain a contradiction as in the proof of Lemma 2.1. This proves (a). To see (b), observe that $w \neq 0$ and, therefore, if $\left\|u_{n}\right\|$ were bounded we would obtain a contradiction from (3.1).

For $\sigma \neq 0$ and $v=\sigma^{-1} u$, equation (2.6) is equivalent to

$$
v=\lambda \varrho\left(\sigma^{-1} F(\sigma v)\right)+w .
$$

With (3.3) we associate the linear problem

$$
v=\lambda m_{\infty} e v+w,
$$

which by the Fredholm alternative has a unique solution $v^{\infty}=v_{k}^{\infty}(\lambda)$, provided

$$
\lambda_{k-1}^{\infty}<\lambda<\lambda_{k}^{\infty}, \quad k=0,1, \ldots,
$$

with $\lambda_{-1}^{\infty}=0$. Furthermore, the Leray-Schauder degree of $v^{\infty}$ is defined and

$$
\operatorname{deg}\left(\text { id }-\lambda m_{\infty} \varrho-w, B\left(v^{\infty}, R\right), 0\right)=\operatorname{deg}\left(\text { id }-\lambda m_{\infty} \ell, B(0, R), 0\right)= \pm 1,
$$

for any $R>0$ and $\lambda \neq \lambda_{i}^{\infty}$. This is a consequence of the homotopy property applied to id $-\lambda m_{\infty} E-t w, t \in[0,1]$ and the fact that id $-\lambda m_{\infty} \mathcal{L}$ is a linear isomorphism. For the ODE-model (2.11) we obtain that

$$
\operatorname{deg}\left(\mathrm{id}-\lambda m_{\infty} \varrho-w, B\left(v_{k}^{\infty}(\lambda), R\right), 0\right)=(-1)^{k}
$$


where $w(x) \equiv 1$, due to the fact that all eigenvalues $\alpha_{i}$ are simple. In Figures 1-4 particular solutions for $\sigma=0$ and the $v_{k}^{\infty}(\lambda)$ are represented by fat dots. The sign \pm attached to them indicates their Leray-Schauder degree.

As a consequence of Lemma 3.1 and (3.5) we have the following lemma.

Lemma 3.2 ( $\sigma$-COntinua). Let $\lambda \neq \lambda_{i}^{\infty}$ be fixed and let $\varepsilon>0$ be given. Then there exists $\sigma_{\infty}>0$ such that:

(a) if $(\lambda, \sigma, v)$ is any solution of (3.3) and $|\sigma|>\sigma_{\infty}$ then $\left\|v-v^{\infty}\right\|<\varepsilon$;

(b) problem (3.3) has a solution for each $\sigma$ with $|\sigma| \geqslant \sigma_{\infty}$.

Proof. First, we have the following inequality for $v \in E \backslash\{0\}$ :

$$
\begin{aligned}
\left\|\lambda \mathscr{L}\left(\sigma^{-1} F(\sigma v)\right)-\lambda m_{\infty} E v\right\| & \leqslant|\lambda|\|\mathcal{L}\|\left\|\sigma^{-1} F(\sigma v)-m_{\infty} v\right\| \\
& =|\lambda|\|\mathcal{L}\|\|v\|\left\|\sigma^{-1} v\right\| o(\|\sigma v\|)=\eta(v, \sigma) .
\end{aligned}
$$

Now, since $E$ is compact (i.e., id $-\lambda m_{\infty} E-w$ is a closed map) and $v^{\infty}$ is a unique solution of (3.4) we have that

$$
\inf \left\{\left\|v-\lambda m_{\infty} \varepsilon v-w\right\|:\left\|v^{\infty}-v\right\| \geqslant \varepsilon\right\}=\delta>0 .
$$

Thus, for $|\sigma|$ sufficiently large, problem (3.3) will not have any solution $v$ with $\left\|v-v^{\infty}\right\| \geqslant \varepsilon$. This proves (a). To see (b), choose $\sigma_{\infty}>0$ such that $\eta(v, \sigma)<\delta / 2$ for all $|\sigma| \geqslant \sigma_{\infty}$ and $v$ such that $\left\|v-v^{\infty}\right\|=\varepsilon$. Define

$$
h(v, t):=v-t \lambda \mathcal{L}\left(\sigma^{-1} F(\sigma v)\right)-(1-t) \lambda m_{\infty} \mathcal{L} v-w .
$$

Then $h(v, t) \neq 0$ for all $t \in[0,1]$ and $\left\|v-v^{\infty}\right\|=\varepsilon$ and, consequently, the homotopy property implies that

$$
\begin{aligned}
\operatorname{deg}\left(\mathrm{id}-\lambda \mathcal{L}\left(\sigma^{-1} F\left(\sigma^{\cdot}\right)\right)-w, B\left(v^{\infty}, \varepsilon\right), 0\right) \\
\quad=\operatorname{deg}\left(\mathrm{id}-\lambda m_{\infty} E-w, B\left(v^{\infty}, \varepsilon\right), 0\right)= \pm 1
\end{aligned}
$$

which proves (b).

The above results may be combined to yield the following global continuation theorem for $\lambda$-sections of the set of all solutions $(\lambda, \sigma, u)$ of problem (2.6).

TheOrem 3.1 ( $\sigma$-CONTINUA). Let $\lambda \neq \lambda_{i}^{\infty}, i=0,1,2, \ldots$, be fixed. Then there exists a continuum $S \subset\{\lambda\} \times \Sigma \times E$, such that:

(a) if $(\lambda, \sigma, u) \in \mathfrak{S}$, then $(\lambda, \sigma, u)$ solves (2.6);

(b) if $\pi_{\Sigma}: \Lambda \times E \rightarrow \Sigma$ is the projection onto $\Sigma$, then $\pi_{\Sigma}(\mathfrak{S})=\Sigma$;

(c) if $\left\{\left(\lambda, \sigma_{n}, u_{n}\right)\right\} \subset S$ and $\left|\sigma_{n}\right| \rightarrow \infty$, then $\sigma_{n}^{-1} u_{n} \rightarrow v^{\infty}$.

Proof. It follows from the above results that there exists an open set $\Theta \subset\{\lambda\} \times \Sigma$ $\times E$, with $\hat{E}_{\sigma}=\{u:(\lambda, \sigma, u) \in \mathcal{\theta}\}$ bounded for each $\sigma \in \Sigma$, such that all solutions of (2.6) are, for each fixed $\sigma$, contained in $\hat{\theta}_{\sigma}$, and

$$
\operatorname{deg}\left(\mathrm{id}-\lambda \varepsilon F-\sigma w, \hat{\ominus}_{\sigma}, 0\right)=\operatorname{deg}\left(\mathrm{id}-\lambda m_{\infty} \varepsilon-w, B\left(v^{\infty}, \varepsilon\right), 0\right) .
$$

Thus, (a) and (b) follow from Lemmata 3.1 and 3.2 together with Theorem 2.2. Finally, if $\left\{\left(\lambda, \sigma_{n}, u_{n}\right)\right\} \subset \delta$ and $\left|\sigma_{n}\right| \rightarrow \infty$, then according to Corollary $3.1,\left\|u_{n}\right\| \rightarrow \infty$ and $\left\{v_{n}=\sigma_{n}^{-1} u_{n}\right\}$ remains bounded. Using equation (3.3) we obtain

$$
v_{n}=\lambda m_{\infty} \varrho v_{n}+\lambda\left\|v_{n}\right\| \mathcal{L}\left(\left\|\sigma_{n} v_{n}\right\|^{-1} o\left(\left\|\sigma_{n} v_{n}\right\|\right)\right)-w .
$$


Thus, $\left\{\boldsymbol{v}_{n}\right\}$ has a convergent subsequence which converges to a solution of problem (3.4). However, (3.4) is uniquely solvable by $v^{\infty}$.

REMARK 3.1. Because of hypothesis (2.2) we may, in fact, assert that for $|\sigma| \gg 1$ the $\sigma$-section $\varsigma_{\sigma}=\{u:(\lambda, \sigma, u) \in \delta\}$ is a single point, and that $\delta$ may be parametrized by $\sigma$ over the intervals $\left[\sigma_{\infty}, \infty\right)$ and $\left(-\infty,-\sigma_{\infty}\right]$, where $\sigma_{\infty} \gg 1$.

So far we have studied $\sigma$-continua for $\lambda \neq \lambda_{i}^{\infty}$ fixed. Now we study $\lambda$-continua for $\sigma$ fixed. We will see that by imbedding the $\lambda$-parameter problem $(1.1)$ into the $(\lambda, \sigma)$ problem (1.3) allows for a new and simple approach to a bifurcation from infinity analysis of problem (1.1). More generally, assume we are given a problem

$$
u-\lambda G(u)=0
$$

in some Banach space $E \ni u$, and $\lambda \in \mathbf{R}$. Let

$$
G(u)=L u+\Phi(u),
$$

where $L$ is compact linear, $\Phi$ is completely continuous and $\|u\|^{-1} \Phi(u) \rightarrow 0$ as $\|u\| \rightarrow \infty$. Furthermore, assume that $\lambda_{\infty}$ is a characteristic value of $L$ of odd multiplicity. Then (3.7) has a bifurcation from infinity at $\lambda_{\infty}$. The by now classical approach to such a problem [14] is to study the transformation $v=\|u\|^{-2} u$. Our approach here is to imbed (3.7) into the two-parameter problem ( $w \in E \backslash\{0\})$,

$$
u=\lambda G(u)+\sigma w .
$$

As an example for (3.7) we investigate (1.1).

Theorem 3.2 ( $\lambda$-CONTINUA). Let $\alpha_{i}$ be an eigenvalue of (2.4) of odd multiplicity and let $\varepsilon>0$ be so small that $\left[\lambda_{i}^{\infty}-\varepsilon, \lambda_{i}^{\infty}+\varepsilon\right]$ does not contain $\lambda_{j}^{\infty}, j \neq i$. Then there exists an $R=R(\varepsilon)$, such that

$$
\left[\lambda_{i}^{\infty}-\varepsilon, \lambda_{i}^{\infty}+\varepsilon\right] \times\{0\} \times(E \backslash B(0, R))
$$

contains an unbounded continuum $\circlearrowright \subset \Lambda \times\{0\} \times E$ of solutions for $(2.6)(\sigma=0)$.

Proof. Firstly, from Lemma 3.1 we may find an $R=R(\varepsilon)>0$, such that if $|\sigma| \leqslant \rho, \rho>0$, and $(\lambda, \sigma, u)$ is a solution of (2.6), $\lambda=\lambda_{i}^{\infty} \pm \varepsilon$, then $\|u\|<R$. Moreover, the discussion in the proof of Lemma 3.2 implies that

$$
\begin{aligned}
& \operatorname{deg}\left(\mathrm{id}-\left(\lambda_{i}^{\infty}-\varepsilon\right) \mathcal{L} F-\sigma w, B(0, R), 0\right)=\operatorname{deg}\left(\mathrm{id}-\left(\lambda_{i}^{\infty}-\varepsilon\right) m_{\infty} \mathcal{L}, B(0, R), 0\right) \text {, } \\
& \operatorname{deg}\left(\mathrm{id}-\left(\lambda_{i}^{\infty}+\varepsilon\right) \mathcal{L} F-\sigma w, B(0, R), 0\right)=\operatorname{deg}\left(\mathrm{id}-\left(\lambda_{i}^{\infty}+\varepsilon\right) m_{\infty} \mathcal{L}, B(0, R), 0\right)
\end{aligned}
$$

for all $|\sigma| \leqslant \rho$. However, since $\alpha_{i}$ is of odd multiplicity it follows (see [13]) that

$$
\operatorname{deg}\left(\mathrm{id}-\left(\lambda_{i}^{\infty}-\varepsilon\right) m_{\infty} \varrho, B(0, R), 0\right) \cdot \operatorname{deg}\left(\mathrm{id}-\left(\lambda_{i}^{\infty}+\varepsilon\right) m_{\infty} \mathcal{L}, B(0, R), 0\right)=-1 \text {. }
$$

Thus, we may apply Theorem 2.3 .

We have seen that the imbedding of problem (1.1) into (1.3) allows an elegant way for the computation of degrees near infinity. Obviously, the behaviour of $f$ near the origin is unimportant for the validity of the result and it is obvius how to establish and prove a result for (3.7). If $L$ is an ordinary differential operator and $f$ satisfies (2.2) then the branches bifurcating from infinity at $\lambda_{i}^{\infty}$ will inherit the nodal structure of the linear problem and, consequently, may not bifurcate from infinity at any other $\lambda_{j}^{\infty}$. To see this one may employ arguments similar to those in $[13,17]$. 
4. Particular $\sigma$-subcontinua. Throughout this section we assume that $f$ satisfies (2.2) and (2.8) and that $\phi(x)>0$ for $x \in \partial \Omega$. Our goal is the investigation of particular $\sigma$-continua of solutions of (2.6). In particular, we want to show the existence of some of the subcontinua which are displayed in Figures 1-4. Our first goal is to show that there is a subcontinuum of solutions of (2.6) for every $\lambda>\max \left\{\lambda_{0}^{0}, \lambda_{0}^{\infty}\right\}$ fixed in $\Sigma \times E$ which connects $\mathscr{P}_{0}(\lambda)$ with $\mathscr{P}_{\infty}(\lambda)\left(\operatorname{resp} . \mathscr{N}_{0}(\lambda)\right.$ with $\left.\mathscr{R}_{\infty}(\lambda)\right)$. We introduce some further notation:

$$
\begin{aligned}
& \mathcal{Q}(\lambda) \subset\{\lambda\} \times \Sigma \times E, \quad \lambda>0 \text { fixed, } \\
& Q(\lambda)=\{(\lambda, \sigma, u) \text { solving }(2.6)\} \text {; } \\
& \Upsilon_{0}^{\circ}(\lambda) \subset\{\lambda\} \times \Sigma \times E, \quad \lambda>0 \text { fixed, } \\
& \widetilde{\Upsilon}_{0}(\lambda)=\left\{(\lambda, \sigma, u): u_{\max }=\max \{u(x): x \in \operatorname{cl} \Omega\}<s_{+}^{0}\right\} \text {; } \\
& \tau^{\infty}(\lambda) \subset\{\lambda\} \times \Sigma \times E, \quad \lambda>0 \text { fixed, } \\
& \Upsilon^{\infty}(\lambda)=\left\{(\lambda, \sigma, u): u_{\max }>s_{+}^{\infty}\right\} \text {; } \\
& \Upsilon_{0}^{\infty}(\lambda) \subset\{\lambda\} \times \Sigma \times E, \quad \lambda>0 \text { fixed, } \\
& \Upsilon_{0}^{\infty}(\lambda)=\left\{(\lambda, \sigma, u): s_{+}^{0}<u_{\max }<s_{+}^{\infty}\right\} ; \\
& \text { 讦 }(\lambda) \subset\{\lambda\} \times \Sigma \times E, \quad \lambda>0 \text { fixed, } \\
& \text { ols }(\lambda)=\{(\lambda, \sigma, u): u(x)>0, x \in \Omega\} \text {; } \\
& \vartheta_{r}^{R}(\lambda)=\{(\lambda, \sigma, u) \in \mathscr{Q}\|(\lambda): r<\| u \|<R\} \text {. }
\end{aligned}
$$

To establish the existence of the desired continua we collect some auxiliary lemmata.

LEMMA 4.1. Let $v$ be a solution of

$$
\begin{aligned}
L v+\beta v=0, & x \in \Omega, \\
v=\phi, & x \in \partial \Omega,
\end{aligned}
$$

and let $\beta>\alpha_{0}$. Then $v$ assumes negative values in $\Omega$.

Proof. Recall that $\phi(x)>0$ for $x \in \partial \Omega$. Assume that $v(x) \geqslant 0$ for all $x \in \Omega$. Thus, by the minimum principle [4, p. 32] we have that $v(x)>0$ for $x \in \Omega$. Since

$$
L v+\alpha_{0} v \leqslant 0, \quad x \in \Omega,
$$

it follows that $v$ is a positive upper solution of

$$
\begin{aligned}
L u+\alpha_{0} u & =0, & & x \in \Omega, \\
u & =\phi, & & x \in \partial \Omega .
\end{aligned}
$$

Furthermore, the trivial solution is a lower solution of (4.8). Hence, (4.8) has a solution $w$ (see Theorem 3.2 of [15]) with $0 \leqslant w \leqslant v$. Again the minimum principle implies that $w(x)>0$ for $x \in \Omega$. Now let $\theta_{0}$ be the positive eigenfunction of (2.4) corresponding to $\alpha_{0}$. We may assume that

$$
0<\theta_{0}(x)<w(x) \text { for } x \in \Omega \text {. }
$$

Let $\mu_{*}=\sup \left\{\mu: w(x)-\mu \theta_{0}(x)>0, x \in \Omega\right\}$, and let

$$
w_{*}=w-\mu_{*} \theta_{0} \text {. }
$$


Then $w_{*}$ is a solution of (4.8) with $w_{*}(x)=0$ for some $x \in \Omega$. Since $w_{*}(x) \geqslant 0$ in $\Omega$ we again get a contradiction to the minimum principle.

REMARK 4.1. If $L$ is the Laplace operator, then the conclusion of Lemma 4.1 is merely a consequence of the strong maximum principle [4, p. 33] and Green's identity: multiply (4.7) by $\theta_{0}$ and integrate to obtain

$$
\int_{\Omega} \Delta v \theta_{0}+\beta \int_{\Omega} v \theta_{0}=0
$$

and then

$$
\int_{\Omega} v \Delta \theta_{0}+\beta \int_{\Omega} v \theta_{0}-\int_{\partial \Omega} \frac{\partial \theta_{0}}{\partial \nu} \phi=0
$$

or

$$
\left(\beta-\alpha_{0}\right) \int_{\Omega} v \theta_{0}=\int_{\partial \Omega} \frac{\partial \theta_{0}}{\partial \nu} \phi,
$$

which implies $v(x)<0$ for some $x$, because $\partial \theta_{0} / \partial \nu<0$.

LEMma 4.2 ( $\sigma$-CONTINUA). Let $\lambda>\lambda_{0}$ be fixed. Then there exists $\sigma_{+}>0$, such that

$$
X^{+}(\lambda)=A(\lambda) \cap\left(W(\lambda) \cup V_{0}^{\infty}(\lambda)\right) \cap\{\lambda\} \times\left[\sigma_{+}, \infty\right) \times E=\varnothing .
$$

Proof. Assume that there exists $\left\{\left(\lambda, \sigma_{n}, u_{n}\right)\right\} \subset X^{+}(\lambda)$ such that $\sigma_{n} \rightarrow \infty$. Then it follows from Theorem 3.1(c) that $\sigma_{n}^{-1} u_{n} \rightarrow v^{\infty}$, where $v^{\infty}$ solves (2.7) with $\lambda m_{\infty}=\beta$ $>\alpha_{0}$. If $u_{n}(x)>0$ for $x \in \Omega$, then $v^{\infty}(x)>0$ for $x \in \Omega$. However, since $\beta>\alpha_{0}$, Lemma 4.1 implies that $v$ assumes negative values and this is a contradiction. If $s_{+}^{0}<\left(u_{n}\right)_{\max }<s_{+}^{\infty}$, then there exists $x \in \Omega$ such that $v^{\infty}(x)=0$ and $v^{\infty}$ has a local maximum at $x$. Hence, $L v^{\infty} \geqslant 0$ in a neighborhood of $x$ and thus, by the maximum principle [4, p. 32] it follows that $v^{\infty} \equiv 0$ in such a neighborhood and, since $v^{\infty}(x)=\phi(x)>0$ for $x \in \partial \Omega$, the minimum principle yields $v^{\infty} \equiv 0$, which again is a contradiction. This proves the existence of $\sigma_{+}$.

Lemma 4.3 ( $\sigma$-CONTINUA). Let $\lambda>\lambda_{0}^{\infty}$ be fixed. Then there exists $\sigma_{-}<0$, such that

$$
X^{-}(\lambda)=A(\lambda) \cap V_{0}^{\infty}(\lambda) \cap\{\lambda\} \times\left(-\infty, \sigma_{-}\right] \times E=\varnothing .
$$

Proof. Assume that there exists $\left\{\left(\lambda, \sigma_{n}, u_{n}\right)\right\} \subset X^{-}(\lambda)$ such that $\sigma_{n} \rightarrow-\infty$. Then it follows from Theorem 3.1(c) that $\sigma_{n}^{-1} u_{n} \rightarrow v^{\infty}$, where $v^{\infty}$ solves (2.7) and $\lambda m_{\infty}=\beta$ $>\alpha_{0}$. Since $s_{+}^{0}<\left(u_{n}\right)_{\max }<s_{+}^{\infty}$ it follows, however, that $v^{\infty}(x) \geqslant 0$ for $x \in \Omega$, contradicting Lemma 4.1 .

Let $\sigma_{+}, \sigma_{-}$be such that (4.9) and (4.10) hold. Define

$$
\begin{aligned}
& \mathcal{U}(\lambda) \subset\{\lambda\} \times \Sigma \times E, \quad \lambda>0 \text { fixed, } \\
& \mathcal{U}(\lambda)=\left\{(\lambda, \sigma, u): u_{\text {min }}=\min \{u(x): x \in \operatorname{cl} \Omega\}<0\right\}
\end{aligned}
$$

and a $\lambda$-section of solutions

$$
\begin{aligned}
\mathscr{F}(\lambda)=\mathscr{Q}(\lambda) \cap\left\{\left(\mathcal{W}_{r}^{R}(\lambda)\right.\right. & \left.\cap\{\lambda\} \times\left[0, \sigma_{+}\right] \times E\right) \\
& \left.\cup\left(\mathcal{V}_{0}^{\infty}(\lambda) \cap \mathcal{U}(\lambda)\right) \times\{\lambda\} \times\left[\sigma_{-}, \sigma_{+}\right] \times E\right\},
\end{aligned}
$$

where $r>0, R>0$ are described later. 
LEMMA 4.4. Let $s>0$ be such that $f(s) \leqslant 0$ and let $(\lambda, \sigma, u) \in \mathscr{F}(\lambda)$ with $\sigma \leqslant 0$. Then

$$
u_{\max }=\max \{u(x): x \in \operatorname{cl} \Omega\} \neq s .
$$

Proof. If $s$ is such that $f(s)<0$ or $f(s)=0$ and $f(s-\varepsilon)<0$ for $\varepsilon \ll 1$, and $u_{\max }=s$, then $u$ assumes its maximum at an interior point $x(\phi(x)>0$ for $x \in \partial \Omega)$, hence

$$
L u=-\lambda f(u) \geqslant 0
$$

in a neighborhood of $x$, which, due to the maximum principle, is impossible unless $u \equiv s$. But since $u(x)=\sigma \phi(x)$ for $x \in \partial \Omega$ and $\sigma \leqslant 0, u$ cannot be constant. If $f(s)=0$ and $f(s-\varepsilon)>0$ for $\varepsilon \ll 1$ define $v \equiv s$ and apply the minimum principle to $v-u$.

We now specify $r>0$ and $R>0$ in (4.12).

LEMMA 4.5 ( $\sigma$-CONTINUA). Let $\lambda \neq \lambda_{i}^{\infty}, i=0,1,2, \ldots$, be fixed. Then there exists $R>0$ such that if

$$
(\lambda, \sigma, u) \in \mathcal{Q}(\lambda) \cap\{\lambda\} \times\left[0, \sigma_{+}\right] \times E \cap\left(\mathcal{V}_{0}^{\infty}(\lambda) \cup \mathcal{Q}(\lambda)\right)
$$

then $\|u\|<R$.

Proof. We may apply Lemma 3.1.

LEMMA 4.6 ( $\sigma$-CONTINUA). Let $\lambda>\lambda_{0}^{0}$ be fixed. Then there exists $r>0$ such that if

$$
(\lambda, \sigma, u) \in \mathbb{Q}(\lambda) \cap\{\lambda\} \times\left[0, \sigma_{+}\right] \times E \cap\left(\mathscr{W}(\lambda) \cup \mathcal{T}_{0}^{\infty}(\lambda)\right)
$$

then $\|u\|>r$.

Proof. There is nothing to prove for $(\lambda, \sigma, u) \in \mathcal{V}_{0}^{\infty}(\lambda)$. Assume that there exists $\left\{\left(\lambda, \sigma_{n}, u_{n}\right)\right\} \subset\{\lambda\} \times\left[0, \sigma_{+}\right] \times E \cap \mathscr{Q}(\lambda) \cap \mathcal{Q}(\lambda)$ and $\left\|u_{n}\right\| \rightarrow 0$. Then $v_{n}=\left\|u_{n}\right\|^{-1} u_{n}$ satisfies

$$
v_{n}=\lambda m_{0} \varrho v_{n}+\lambda \varrho\left(\left\|u_{n}\right\|^{-1} o\left(u_{n}\right)\right)+\left\|u_{n}\right\|^{-1} \sigma_{n} w
$$

Thus, $\left\|u_{n}\right\|^{-1} \sigma_{n}$ is bounded and we may assume that $\left\|u_{n}\right\|^{-1} \sigma_{n} \rightarrow \gamma \geqslant 0$. Moreover, we obtain that $\left\{v_{n}\right\}$ has a convergent subsequence converging to $v$. Observe that $v(x)>0$ for $x \in \Omega$ and that

$$
\begin{aligned}
L v+\lambda m_{0} v=0, & x \in \Omega, \\
v=\gamma \phi, & x \in \partial \Omega,
\end{aligned}
$$

we thus obtain a contradiction to Lemma 4.1, because $\lambda m_{0}>\alpha_{0}$.

We may summarize our considerations above in Figure 6, where dark shaded regions represent regions in $\{\lambda\} \times \Sigma \times E$, which have empty intersection with $\mathscr{F}(\lambda)$. 


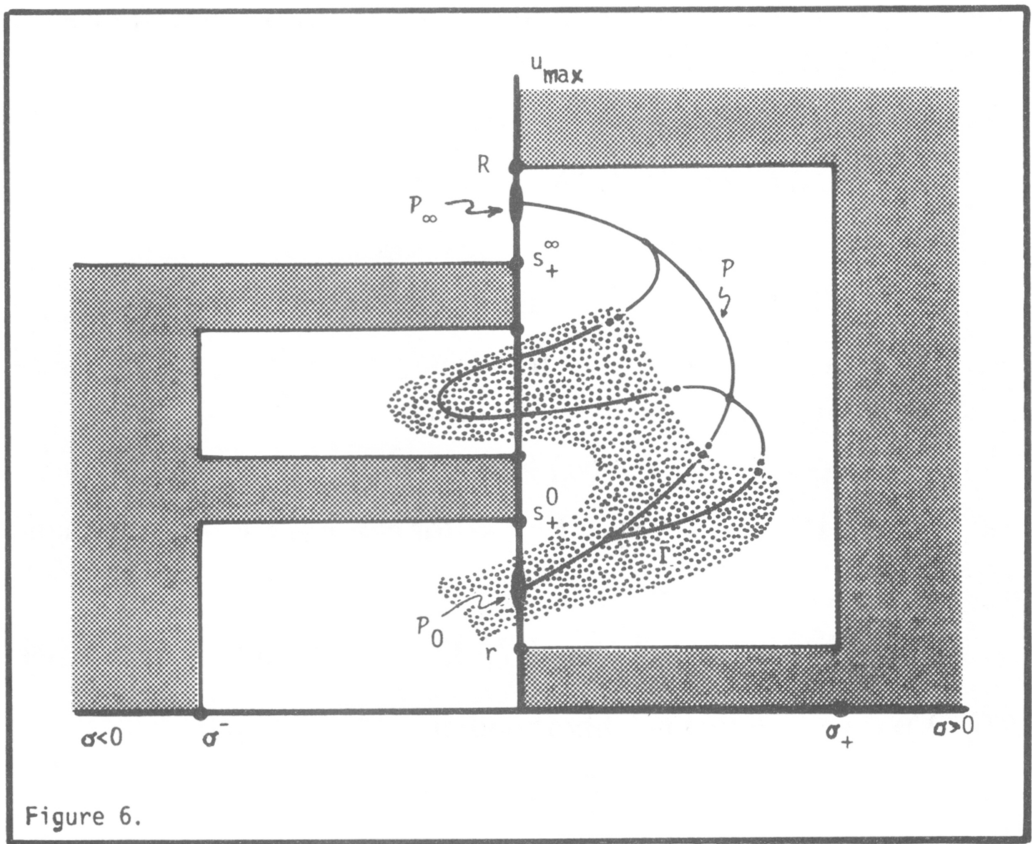

The goal is now to use the 'a priori' bounds on solutions in $\mathscr{F}(\lambda)$ to establish a continuum from $\mathscr{P}_{0}(\lambda)$ to $\mathscr{P}_{\infty}(\lambda)$ (see also Figures $\left.2-4\right)$.

Theorem 4.1 ( $\sigma$-Continua). Let $\lambda>\max \left\{\lambda_{0}^{0}, \lambda_{0}^{\infty}\right\}$ and $\lambda \neq \lambda_{i}^{\infty}$ be fixed. Then there exists a continuum $\mathscr{P}(\lambda) \subset \mathcal{F}(\lambda)$ such that

$$
\mathscr{P}(\lambda) \cap \mathscr{P}_{0}(\lambda) \neq \varnothing \neq \mathscr{P}(\lambda) \cap \mathscr{P}_{\infty}(\lambda) .
$$

Proof. We first argue that $\mathscr{F}(\lambda)$ is a compact subset of $\mathscr{Q}(\lambda)$. Since $\mathscr{F}(\lambda)$ is bounded it is certainly relatively compact as the image of a completely continuous operator. Thus, it suffices to show that $\mathscr{F}(\lambda)$ is closed in $\mathscr{Q}(\lambda)$. To see this, observe that

$$
\begin{aligned}
\mathscr{F}(\lambda) \subset \mathscr{Q}(\lambda) \cap[\{\lambda\} \times[0, \infty) \times E \cup \\
\left.\quad\left\{\operatorname{cl}_{0}^{\infty}(\lambda) \cap \operatorname{cl} \mathcal{U}(\lambda) \cap\{\lambda\} \times(-\infty, 0] \times E\right\}\right]=\mathscr{B}(\lambda)
\end{aligned}
$$

and that the set $\mathscr{B}(\lambda)$ is closed in $\mathscr{Q}(\lambda)$. Then the compactness of $\mathscr{F}(\lambda)$ follows, because $\mathscr{F}(\lambda)$ is isolated in $\mathscr{B}(\lambda)$. To obtain $\mathscr{P}(\lambda)$, we argue in the spirit of the Leray-Schauder continuation principle since $\mathscr{P}_{0}(\lambda)$ and $\mathscr{P}_{\infty}(\lambda)$ are nonempty closed subsets of $\mathscr{F}(\lambda)$. Hence, using Lemma 2.1 it suffices to show that assuming that $\mathscr{F}(\lambda)=K_{0} \cup K_{\infty}$ with

$$
K_{0} \cap K_{\infty}=\varnothing, \quad \mathscr{P}_{0}(\lambda) \subset K_{0}, \quad \mathscr{P}_{\infty}(\lambda) \subset K_{\infty}
$$

leads to a contradiction. This contradiction will be obtained by employing the generalized homotopy property of degree and Lemma 2.2. Hence, assume that closed 
sets $K_{0}$ and $K_{\infty}$, with the above properties, exist. Then we may find an open and bounded subset $\Gamma \subset\{\lambda\} \times \Sigma \times E$ such that $K_{0} \subset \Gamma$,

$$
\begin{gathered}
\mathscr{Q}(\lambda) \cap \partial \Gamma \subset\{\lambda\} \times(-\infty, 0] \times\left\{u \in E: u_{\max } \leqslant s_{+}^{0} \text { or } u_{\max } \geqslant s_{+}^{\infty}\right\}, \\
\mathbb{Q}(\lambda) \cap \partial \Gamma \cap\{\lambda\} \times[0, \infty) \times E=\varnothing, \\
\mathbb{Q}(\lambda) \cap \Gamma \cap\{\lambda\} \times\{0\} \times E \\
=\{\lambda\} \times\{0\} \times\left\{u \in E: u(x)>0, x \in \Omega, \text { or } s_{+}^{0}<u_{\max }<s_{+}^{\infty}\right\} .
\end{gathered}
$$

Furthermore, $\Gamma \cap\{\lambda\} \times\{\sigma\} \times E=\varnothing$ for $\sigma>\sigma_{+}$and $\sigma<\sigma_{-}$. Let us abbreviate the compact vectorfield id $-\lambda £ F-\sigma w=G_{\sigma}$. Then,

$$
\operatorname{deg}\left(G_{\sigma}, \Gamma \cap\{\lambda\} \times\{\sigma\} \times E, 0\right) \equiv d_{1} \text { for all } \sigma \geqslant 0,
$$

and $d_{1}=0$, because $\Gamma \cap\{\lambda\} \times\{\sigma\} \times E=\varnothing$ for $\sigma>\sigma_{+}$. On the other hand,

$$
\begin{aligned}
& \operatorname{deg}\left(G_{0}, \Gamma \cap\{\lambda\} \times\{0\} \times E, 0\right) \\
& \quad=\operatorname{deg}\left(G_{0}, U\left(\mathscr{P}_{0}(\lambda)\right), 0\right)+\operatorname{deg}\left(G_{0}, \Gamma \cap \mathcal{V}_{0}^{\infty} \cap\{\lambda\} \times\{0\} \times E, 0\right),
\end{aligned}
$$

where $U\left(\mathscr{P}_{0}(\lambda)\right)$ is an isolating neighborhood of $\mathscr{P}_{0}(\lambda)$ in $E$ and

$$
\operatorname{deg}\left(G_{0}, U\left(\mathscr{P}_{0}(\lambda)\right), 0\right)=+1 .
$$

However,

$$
\operatorname{deg}\left(G_{\sigma}, \Gamma \cap \mathcal{T}_{0}^{\infty} \cap\{\lambda\} \times\{\sigma\} \times E, 0\right) \equiv d_{2}
$$

for all $\sigma \leqslant 0$ and $d_{2}=0$, because $\Gamma \cap \mathcal{V}_{0}^{\infty}(\lambda) \times\{\lambda\} \times\{\sigma\} \times E=\varnothing$ for $\sigma<\sigma_{-}$. Thus we arrive at a contradiction.

REMARK 4.2. In Figure 6 we indicate two possible continua for $\mathscr{P}(\lambda)$. Without further assumptions on $f$ we cannot exclude that $\mathscr{P}(\lambda)$ has nontrivial sections in $\mathcal{V}_{0}^{\infty}(\lambda) \cap\{\lambda\} \times\{\sigma\} \times E$ for $\sigma<0$. Indeed, Brown and Budin [3] and Hess [5] have proved the existence of solutions to (2.6) for $\sigma=0$ and $\lambda$ sufficiently large so that $s_{+}^{0}<\|u\|<s_{+}^{\infty}$. It is unknown to us whether $\mathcal{P}(\lambda)$ will, in fact, pass through these solutions, whenever they exist. Our numerical experiments seem to indicate that this is indeed so.

REMARK 4.3. Due to assumption (2.8) our problem (2.3), or equivalently (2.6), will also have a continuum

$$
\begin{aligned}
& \mathscr{T}(\lambda) \subset\left(\left\{(\lambda, \sigma, u): \lambda>0 \text { fixed, } \sigma \in\left[\sigma_{-}^{*}, 0\right], u(x)<0, x \in \Omega\right\}\right. \\
& \cup\{(\lambda, \sigma, u): \lambda>0 \text { fixed, } \\
& \left.\left.\quad \sigma \in\left[\sigma_{-}^{*}, \sigma_{+}^{*}\right], s_{-}^{\infty}<u_{\min }<s_{-}^{0} \text { and } u_{\max }>0\right\}\right) \cap \mathbb{Q}(\lambda)
\end{aligned}
$$

such that

$$
\Re(\lambda) \cap \mathscr{K}_{0}(\lambda) \neq \varnothing \neq \mathscr{K}(\lambda) \cap \mathscr{K}_{\infty}(\lambda)
$$

for all $\lambda>\max \left\{\lambda_{0}^{0}, \lambda_{0}^{\infty}\right\}$ and $\lambda \neq \lambda_{i}^{\infty}$ fixed. (See Lemma 2.2 for notation.) Here $\sigma_{-}^{*}<0$ and $\sigma_{+}^{*}>0$ are constants which are guaranteed by results analogous to Lemmata 4.2 and 4.3. Figures 2-4 display $\mathscr{P}(\lambda)$ and $\mathcal{N}(\lambda)$ for different choices of $\lambda$ assuming that there are no solutions as discussed in Remark 4.2, i.e. $\mathscr{P}(\lambda)$ has no 
nontrivial $\sigma$-sections for $\sigma<0$, and $\Re(\lambda)$ has no nontrivial $\sigma$-sections for $\sigma>0$. If (2.8) is not satisfied but

$$
0<s_{+}^{0}<s_{+}^{\infty}<\infty
$$

or alternatively

$$
-\infty<s_{-}^{\infty}<s_{-}^{0}<0
$$

then $\mathscr{P}(\lambda)$, or alternatively $\mathscr{N}(\lambda)$, would still exist according to our previous argument.

We have seen in Theorem 4.1 and Remark 4.3 that the set of all solutions of the two-parameter problem (2.6) has structural properties which are independent of $\lambda$ if we look at $\lambda$-sections. These are the subcontinua $\mathscr{P}(\lambda)$ and $\mathscr{N}(\lambda)$. In our next results we discuss the $\lambda$-sections for $\|u\|$ sufficiently small and $\|u\|$ sufficiently large.

For fixed $\lambda>0$ let

$$
\begin{aligned}
& \mathfrak{K}_{0}^{+}(\lambda)=\left\{(\lambda, 0, u) \in \mathbb{Q}(\lambda): 0<u_{\max }<s_{+}^{0}\right. \text { and } \\
& \mathfrak{K}_{0}^{0}(\lambda)=\left\{(\lambda, 0, u) \in \mathbb{Q}(\lambda): s_{-}^{0}<u_{\text {min }}<0\right. \text { and } \\
& \left.\mathfrak{M}_{-}^{0}<u(x)<s_{+}^{0}, x \in \Omega\right\}, \\
& \mathfrak{K}_{\infty}^{+}(\lambda)=\left\{(\lambda, 0, u) \in \mathbb{Q}(\lambda): u_{\text {max }}>s_{+}^{\infty}\right\}, \\
& \mathfrak{K}_{\infty}^{-}(\lambda)=\left\{(\lambda, 0, u) \in \mathbb{Q}(\lambda): u_{\text {min }}<s_{-}^{\infty}\right\} .
\end{aligned}
$$

We wish to demonstrate the existence of a continuum in $\{\lambda\} \times \Sigma \times E$ connecting $\mathfrak{R}_{0}^{+}$and $\mathfrak{K}_{0}^{-}$with $\{\lambda\} \times\{0\} \times\{0\}$. To this end we first observe the following lemma.

LEMMA 4.7. Let $\lambda \neq \lambda_{i}^{0}, i=0,1,2, \ldots$ and let $U_{\varepsilon}\left(\Re_{0}^{+}(\lambda)\right)$ and $U_{\varepsilon}\left(\Re_{0}(\lambda)\right)$ be isolating $\varepsilon$-neighborhoods of $\Re_{0}^{+}(\lambda)$ and $\Re_{0}(\lambda)$. Then

$$
-\operatorname{deg}\left(\mathrm{id}-\lambda \varrho F, U_{\varepsilon}\left(\Re_{0}^{+}(\lambda)\right), 0\right)=\operatorname{deg}\left(\mathrm{id}-\lambda \varrho F, B_{\varepsilon}(0), 0\right),
$$

where $B_{\varepsilon}(0)$ is an isolating $\varepsilon$-neighborhood of $\{\lambda\} \times\{0\} \times\{0\}$.

Proof. Let $R=\left\{(\lambda, 0, u): s_{-}^{0}<u(x)<s_{+}^{0}, x \in \Omega\right\}$. Then we may conclude from Lemma 4.4 and the homotopy property that

$$
\operatorname{deg}(\mathrm{id}-\lambda £ F, R, 0)=+1
$$

and, by the additivity property,

$$
\begin{aligned}
& \operatorname{deg}(\mathrm{id}-\lambda \varrho F, R, 0)=\operatorname{deg}\left(\mathrm{id}-\lambda \varrho F, B_{\varepsilon}(0), 0\right) \\
& \quad+\operatorname{deg}\left(\mathrm{id}-\lambda \varrho F, U_{\varepsilon}\left(\Re_{0}^{+}(\lambda)\right), 0\right)+\operatorname{deg}\left(\mathrm{id}-\lambda \varrho F, U_{\varepsilon}\left(\Re_{0}(\lambda)\right), 0\right) .
\end{aligned}
$$

Thus, the result follows from Lemma 2.2.

Theorem 4.2 ( $\sigma$-CONTINUA). Let $\lambda \neq \lambda_{i}^{0}$ and $\lambda \neq \lambda_{i}^{\infty}, i=0,1,2, \ldots$, and $\lambda>\lambda_{0}^{0}$ fixed. Then there exists a continuum $\Theta(\lambda)$ with

$$
\begin{gathered}
\vartheta(\lambda) \subset \mathscr{Q}(\lambda) \cap\left\{(\lambda, \sigma, u): \sigma<0 \text { and } 0<u_{\max }<s_{+}^{0}\right\} \cup \Re_{0}^{+}(\lambda) \cup\{\lambda, 0,0\}, \\
\{(\lambda, 0,0)\} \in \vartheta(\lambda) \text { and } \vartheta(\lambda) \cap \Re_{0}^{+}(\lambda) \neq \varnothing .
\end{gathered}
$$


Proof. Again our proof will be a combination of Lemma 2.1, appropriate 'a priori' bounds and the Leray-Schauder continuation principle: We first note that there must exist $\sigma_{-}<0$, such that whenever $(\lambda, \sigma, u) \in \mathbb{Q}(\lambda)$ and $0<u_{\max }<s_{+}^{0}$, then $\sigma \geqslant \sigma_{-}$. This follows by much the same arguments as used for the proof of Lemmata 4.2 and 4.3, exploiting Lemma 4.1 and Theorem 3.1 once again. We may summarize these considerations and Lemma 4.4 in Figure 7, where the shaded regions represent regions in $\{\lambda\} \times \Sigma \times E$, which have empty intersection with $\mathscr{Q}(\lambda)$.

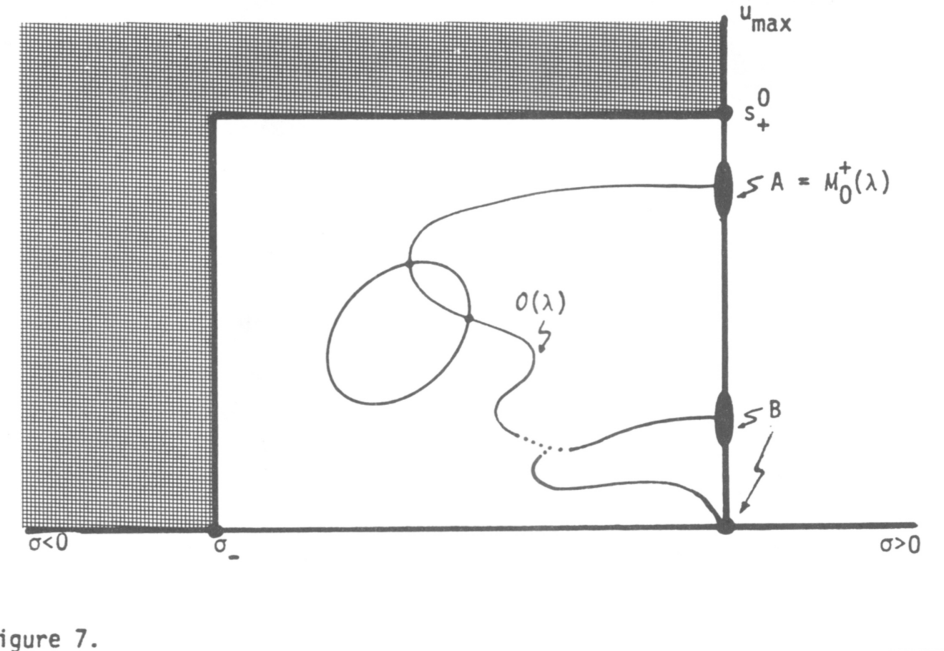

Since $\operatorname{deg}\left(\mathrm{id}-\lambda \mathscr{L} F, U_{\varepsilon}\left(\Re_{0}^{+}(\lambda)\right), 0\right) \neq 0$ by Lemma $4.7 \quad\left(\lambda \neq \lambda_{i}^{0}\right.$ implies that $\operatorname{deg}\left(\mathrm{id}-\lambda \varrho F, B_{\varepsilon}(0), 0\right)= \pm 1$ by the Leray-Schauder formula [7]) we may conclude that there exists a continuum $\vartheta(\lambda) \subset \mathbb{Q}(\lambda)$ such that

(a) $\theta(\lambda) \cap \Re_{0}^{+}(\lambda) \neq \varnothing$,

(b) $(\lambda, \sigma, u) \in \theta(\lambda), \quad \sigma<0 \Rightarrow \sigma>\sigma_{-}$and $0<u_{\max }<s_{+}^{0}$,

(c) $\left(\lambda, 0, u^{*}\right) \in \Theta(\lambda) \backslash \Re_{0}^{+}(\lambda)$ for some $u^{*}$.

Indeed, letting $V(\lambda)$ be the open set (in $\{\lambda\} \times\{0\} \times E)$,

$$
V(\lambda)=R \backslash\left\{U_{\varepsilon}\left(\Re_{0}^{+}(\lambda)\right) \cup U_{\varepsilon}\left(\Re_{0}(\lambda)\right)\right\},
$$

then

$$
\operatorname{deg}(\mathrm{id}-\lambda \varrho F, V(\lambda), 0)=\operatorname{deg}\left(\mathrm{id}-\lambda \varrho F, B_{\varepsilon}(0), 0\right),
$$

where $R$ is defined as in the proof of Lemma 4.7. Thus, letting

$$
A=\Re_{+}^{0}(\lambda) \text { and } B=Q(\lambda) \cap V(\lambda)
$$

we have that $A \neq \varnothing \neq B$ and we can argue as in the proof of Theorem 4.1 to obtain, with the aid of Lemma 2.1 and the generalized homotopy property of degree, 
$\Theta(\lambda)$ satisfying (a) and (c). To see (b), assume that there is a sequence $\left\{\left(\lambda, \sigma_{n}, u_{n}\right)\right\} \subset$ $\mathcal{O}(\lambda)$ with $\sigma_{n} \leqslant 0$ and $\left(u_{n}\right)_{\max }>0$ and such that $\lim \sigma_{n}=\sigma<0$ and $\lim u_{n}=u$ with $u_{\max } \ngtr 0$. Then $u_{\max }=0$, which yields a contradiction to the maximum principle. This proves (4.14). To prove (4.15) we must show that $B=\{(\lambda, 0,0)\}$. Thus, assume that there is a sequence $\left\{\left(\lambda, \sigma_{n}, u_{n}\right)\right\} \subset \mathcal{O}(\lambda)$ with $\sigma_{n}<0$ and $\lim \sigma_{n}=0$, and $\left(u_{n}\right)_{\max }>0$ and $\lim u_{n}=u^{*}$, such that $u^{*} \not \equiv 0$ and $\left(\lambda, 0, u^{*}\right) \notin \mathscr{N}_{+}^{0}(\lambda)$. Since $\sigma=0, u^{*}$ solves (2.3) with $u^{*}(x)=0, x \in \partial \Omega$. Now, if $u^{*}(x)=0$ for some $x \in \Omega$ we would have a contradiction to the maximum principle. If $u^{*}(x)<0$ for all $x \in \Omega$, let $x_{n} \in \Omega$ be a sequence such that $u_{n}\left(x_{n}\right)=\left(u_{n}\right)_{\max }$. Then $\nabla u_{n}\left(x_{n}\right)=0$ and we may assume that $\left\{x_{n}\right\}$ converges to $x_{0}$. Since $u^{*}(x)<0$ we may conclude that $x_{0} \in \partial \Omega$. Finally, we have $C^{1}$-convergence of $\left\{u_{n}\right\}$ and, therefore, we may conclude that $\nabla u^{*}\left(x_{0}\right)=0$. Thus,

$$
\frac{\partial u^{*}}{\partial \nu}\left(x_{0}\right)=0
$$

where $\nu$ is the outer normal of $\partial \Omega$, and this is in contradiction to the strong maximum principle (cf. [4, p. 33]).

Our next goal is to discuss a particular example (see (4.19)) for which we can show that Figure 8 is valid for all $\lambda^{*}=\lambda_{0}^{0}-\varepsilon, 0<\varepsilon \ll 1$, and that Figure 9 is valid for all $\lambda^{*}=\lambda_{0}^{0}+\varepsilon, 0<\varepsilon \ll 1$ (see also Figures 1, 2).

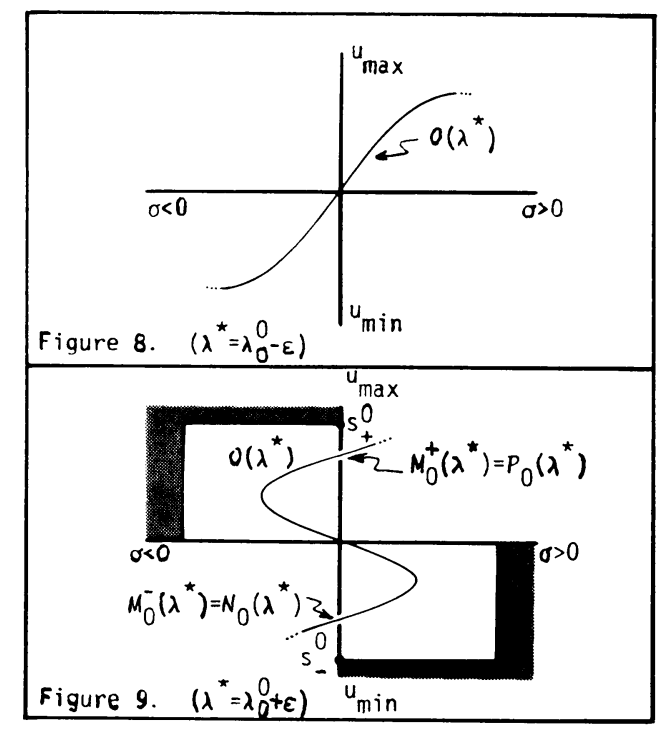

The reason for our interest in these particular $\sigma$-continua is that they constitute an unfolding of a pitchfork bifurcation of the homogeneous problem (2.6) in $\Lambda \times\{0\} \times$ $E$ at $\lambda_{0}^{0}$. Figure 10 shows a cusp in $\Lambda \times \Sigma \times E$ of solutions of the two-parameter problem (2.6) which allows the interpretation that Figures 8 and 9 are just $\lambda$-sections of this singularity surface. 


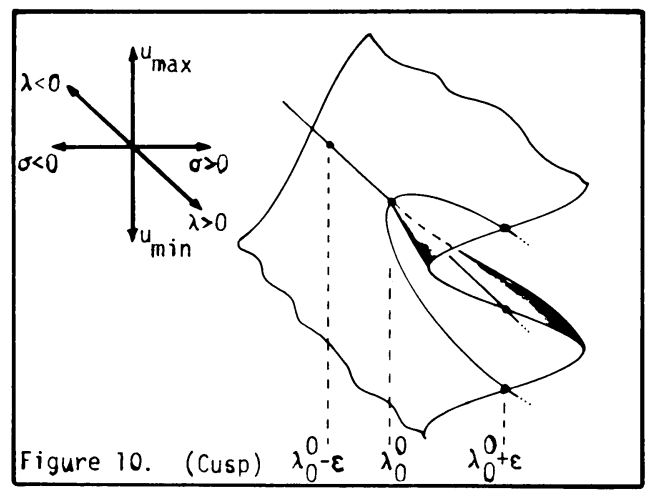

For this interpretation we have to show

$\Re_{+}^{0}\left(\lambda^{*}\right)=$ a unique positive solution, $\quad \lambda^{*}=\lambda_{0}^{0}+\varepsilon$,

$\Re_{-}^{0}\left(\lambda^{*}\right)=$ a unique negative solution, $\quad \lambda^{*}=\lambda_{0}^{0}+\varepsilon ;$ (for $\lambda^{*}=\lambda_{0}^{0}-\varepsilon$ there exists a continuum $\Theta^{*}\left(\lambda^{*}\right) \subset\left\{\lambda^{*}\right\} \times \Sigma$ $\times E$ of solutions for (2.6), such that if $\left(\lambda^{*}, \sigma, u\right) \in \mathcal{E}\left(\lambda^{*}\right)$ and $\sigma>0$, then $u(x)>0, x \in \Omega ;\left(\lambda^{*}, \sigma, u\right) \in \mathcal{E}\left(\lambda^{*}\right)$ and $\sigma<0$, then $u(x)<0, x \in \Omega$.

Then Theorem 4.2 together with (4.17) constitute Figure 9, because Theorem 4.2 has the obvious analogue, that $\Re_{-}^{0}\left(\lambda^{*}\right), \lambda^{*}>\lambda_{0}^{0}$, may via positive $\sigma$ be connected to $(\lambda, 0,0)$. Furthermore (4.18) constitutes Figure 8. Our further assumptions on problem (2.3), which permit us to show (4.17) and (4.18), are

$$
L u=\Delta u ; \quad f \in C^{\infty} \text { and } f(s) / s<m_{0} \text { for } s_{-}^{0}<s<s_{+}^{0}, s \neq 0 .
$$

The following lemma will be useful.

LEMMA 4.8. Let $(\lambda, \sigma, u)$ be a solution of (2.3). Furthermore, let $\Omega_{p} \subset \operatorname{cl} \Omega$ (possibly $\Omega_{p}=\Omega$ ) be such that

$$
\begin{aligned}
& u(x)>0, \quad x \in \Omega_{p}, \\
& u(x)=0, \quad x \in \partial \Omega_{p},
\end{aligned}
$$

Then $\lambda>\lambda_{0}^{0}$.

Proof. (Assume $\Omega_{p} \neq \Omega$, the other case may be treated similarly.) Note that $u$ satisfies the equation

$$
\begin{aligned}
\Delta u+\lambda(f(u) / u) u & =0, \quad x \in \Omega_{p}, \\
u & =0, \quad x \in \partial \Omega_{p} .
\end{aligned}
$$

Let $v$ be a positive eigenfunction of $\Delta$ corresponding to $\alpha_{0}=\lambda_{0}^{0} m_{0}$, i.e.

$$
\begin{aligned}
\Delta v+\alpha_{0} v & =0, & & x \in \Omega, \\
v & =0, & & x \in \partial \Omega .
\end{aligned}
$$


Hence,

$$
\int_{\Omega_{p}}[\Delta v u-\Delta u v]+\int_{\Omega_{p}}\left[\alpha_{0}-\lambda f(u) / u\right] u v=0,
$$

i.e. by Green's identity ( $\nu$ the outer normal of $\partial \Omega_{p}$ )

$$
-\int_{\partial \Omega_{p}}\left[v \frac{\partial u}{\partial \nu}-u \frac{\partial v}{\partial \nu}\right]+\int_{\Omega_{p}}\left[\alpha_{0}-\lambda f(u) / u\right] u v=0 .
$$

Note that

$$
\int_{\partial \Omega_{p}}\left[v \frac{\partial u}{\partial \nu}-u \frac{\partial v}{\partial \nu}\right]<0
$$

because $u(x)=0, x \in \partial \Omega_{p}$ and $\partial u(x) / \partial \nu<0, x \in \partial \Omega_{p}$, due to the strong maximum principle (see [4, p. 33]). Thus $\lambda>\lambda_{0}^{0}$.

We now prove (4.18). First, we note that if $\lambda^{*}=\lambda_{0}^{0}-\varepsilon, 0<\varepsilon \ll 1$, then (Leray-Schauder formula)

$$
\operatorname{deg}\left(\mathrm{id}-\lambda * \varrho F, B_{\delta}(0), 0\right)= \pm 1,
$$

and, therefore, the solution $\left(\lambda^{*}, 0,0\right)$ can be continued due to the Leray-Schauder continuation principle on a compact $\sigma$-interval in $\left\{\lambda^{*}\right\} \times \Sigma \times E$. This provides a continuum $\theta\left(\lambda^{*}\right)$ for which we need to verify (4.18). Assume that there exists $\left(\lambda^{*}, \sigma, u\right) \in \Theta\left(\lambda^{*}\right)$ with

$$
\lambda^{*}=\lambda_{0}^{0}-\varepsilon, \quad \sigma<0, \quad u(x) \nless 0, \quad x \in \Omega .
$$

Let $\Omega_{p} \subset \Omega$ be the domain such that

$$
\begin{aligned}
& u(x)>0, \quad x \in \Omega_{p}, \\
& u(x)=0, \quad x \in \partial \Omega_{p} .
\end{aligned}
$$

We may apply Lemma 4.8 to obtain a contradiction. The argument for $\sigma>0$ is analogous. This proves (4.18). To show (4.17) we recall that due to the maximum principle and the Krein-Rutman Theorem $\alpha_{0}$, the principal eigenvalue of $\Delta$ with respect to $\Omega$, is simple and therefore we may apply a theorem of Crandall and Rabinowitz [13] to conclude that the bifurcation from zero, for problem (2.6) in $\Lambda \times\{0\} \times E$ at $\lambda_{0}^{0}=\alpha_{0} m_{0}$, is locally given by smooth curves of positive and negative solutions, which can be parametrized over $\lambda$ and which are locally unique. Thus, it suffices to show that:

These curves constitute a supercritical pitchfork bifurcation, (see Figure 11)

and

$$
\text { that if }(\lambda, 0, u) \text { is a solution of (2.6) with } \lambda \leqslant \lambda_{0}^{0} \text { and }
$$
$s_{-}^{0}<u(x)<s_{+}^{0}$, then $u \equiv 0$. 


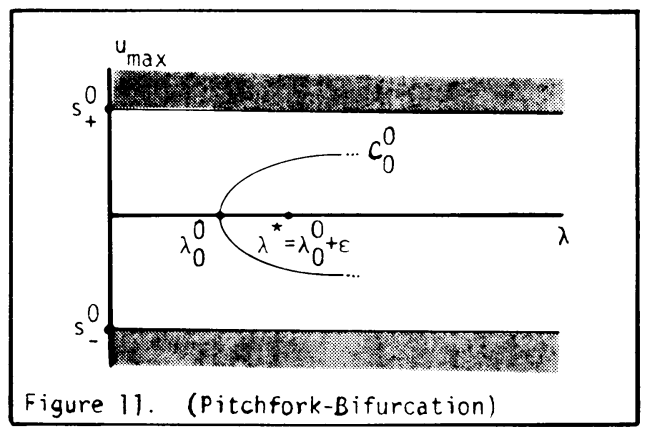

To show (4.21) suppose there is a positive solution $(\lambda, 0, u), \lambda \leqslant \lambda_{0}^{0}$, with $0<u(x)$ $<s_{+}^{0}, x \in \Omega$. Then we may apply Lemma 4.8 with $\Omega=\Omega_{p}$ to obtain a contradiction. The argument for negative solutions is analogous. To show (4.22) suppose there is a solution $(\lambda, 0, u)$ of $(2.6)$ with $\lambda \leqslant \lambda_{0}^{0}$ and $u(x)$ changes sign in $\Omega$. Then, again we may apply Lemma 4.8 to obtain a contradiction. This proves (4.17).

REMARK 4.4. It is noteworthy that we have obtained an unfolding interpretation for problem (2.6) without imposing any conditions of $f^{\prime \prime}$ and $f^{\prime \prime \prime}$ as seems to be necessary in a singularity theory approach.

Similarly to the above discussion we also can characterize solutions $(\lambda, \sigma, u)$ for $|\sigma|$ large and $\|u\|$ large. Let $\mathcal{S}(\lambda)$ be the $\sigma$-continuum of Theorem 3.1.

TheOrem 4.3 ( $\sigma$-CONTINUA). Let $\lambda>\lambda_{0}^{\infty}$ and let $\lambda \neq \lambda_{i}^{\infty}, i=1,2,3, \ldots$ Then there exists a subcontinuum ${ }^{2}{ }^{+}(\lambda) \subset \varsigma(\lambda)$ of solutions in $\{\lambda\} \times[0, \infty) \times E$ such that

$$
\pi_{\Sigma}\left(\mathfrak{Q}^{+}(\lambda)\right)=[0, \infty) \text { and } \mathfrak{Q}^{+}(\lambda) \cap\{\lambda\} \times\{0\} \times E \subset \mathfrak{M}_{\infty}^{-}(\lambda) \text {. }
$$

Furthermore, if $U_{\varepsilon}$ is an isolating neighborhood of $\mathfrak{M}_{\infty}^{-}(\lambda)$ in $E$, then

$$
\left.\operatorname{deg}\left(\mathrm{id}-\lambda E F, U_{\varepsilon}()_{\infty}^{-}(\lambda)\right), 0\right)=\operatorname{deg}\left(\mathrm{id}-\lambda m_{\infty} E, B_{\varepsilon}\left(v^{\infty}\right), 0\right) .
$$

Proof. We have shown in Theorem 3.1 that if $\left(\lambda, \sigma_{n}, u_{n}\right) \in S(\lambda)$ and $\sigma_{n} \rightarrow \infty$ then $\sigma_{n}^{-1} u_{n} \rightarrow v^{\infty}$. Hence, due to Lemma 4.1, $\sigma_{n}^{-1} u_{n}$ must have a negative minimum in $\Omega$ and consequently $u_{\min }<s_{-}^{\infty}$ for $\sigma_{n} \gg 1$. Now it follows from Lemma 4.4 that (2.6) may not have any solution with $u_{\min }=s_{-}^{\infty}$. Hence, $\delta(\lambda)$ will have a subcontinuum with the desired properties and the degree computation is a consequence of the homotopy property.

In a completely analogous way one proves the existence of a subcontinuum $\mathcal{Q}^{-}(\lambda) \subset \delta(\lambda)$ such that

$$
\pi_{\Sigma}\left(\mathcal{Q}^{-}(\lambda)\right)=(-\infty, 0], \quad \mathcal{Q}^{-}(\lambda) \cap\{\lambda\} \times\{0\} \times E \subset \mathfrak{M}_{\infty}^{+}(\lambda) .
$$

These constitute the $\sigma$-continua in Figures $2-4$. In particular, in Figure 3 it is apparent that one should not think of $\mathcal{2}^{+}(\lambda)$ as a single curve. Also note that $\mathcal{Q}^{+}(\lambda) \cap\{\lambda\} \times\{0\} \times E$ is not a single point. Summarizing we have the following "universal" subcontinua in $\mathcal{S}(\lambda)$ :

$(\alpha)$ connections from $\mathscr{P}_{0}(\lambda)$ to $\mathscr{P}_{\infty}(\lambda)$ and $\mathscr{K}_{0}(\lambda)$ to $\mathcal{K}_{\infty}(\lambda)$,

$(\beta)$ connections from $\mathfrak{K}_{0}^{+}(\lambda)$ and $\mathfrak{K}_{0}^{-}(\lambda)$ to $(\lambda, 0,0)$, 
$(\gamma)$ connections from $\mathfrak{M}_{\infty}^{+}(\lambda)$ and $\mathfrak{R}_{\infty}^{-}(\lambda)$ to $\left(\lambda, \sigma^{\infty}, u^{\infty}\right)$, where

$$
\left|\sigma^{\infty}\right| \gg 1 \text { and }\left\|\left(\sigma^{\infty}\right)^{-1} u^{\infty}-v^{\infty}\right\| \ll 1 .
$$

This leads to the view that we can, in a quite natural and obvious way, distinguish an "inner world" in $Q(\lambda)$ corresponding to $(\beta)$ from an "outer world" in $Q(\lambda)$ corresponding to $(\gamma)$. In this regard the continua of $(\alpha)$ serve as a connection from the "inner" to the "outer world". In $\$ 5$ we will see that the "symmetries" between the "inner" and "outer world" are much richer.

One possibility to establish a more rigorous correspondence between the "inner" and "outer world" is by analyzing the transformation

$$
E \backslash\{0\} \ni u \mapsto v=u\|u\|^{-2},
$$

the reflection with respect to the unit sphere. Let $\lambda \neq \lambda_{i}^{0}, \lambda \neq \lambda_{i}^{\infty}, i=0,1,2, \ldots$, and let $(\lambda, \sigma, u)$ be a solution of (2.6). Then $v$ satisfies the equation

$$
v=\lambda \mathcal{L}\left(\|v\|^{2} F\left(v\|v\|^{-2}\right)\right)+\sigma \cdot\|v\|^{-2} w .
$$

Now let $(\lambda, \sigma, u)$ lie on the continuum $\mathscr{C}(\lambda)$ whose existence was established in Theorem 4.2. Then, as $\sigma \rightarrow 0$ we have that either $\|u\| \rightarrow 0$ or that $(\lambda, \sigma, u) \rightarrow$ $\left(\lambda, 0, u^{*}\right) \in \mathfrak{M}_{0}^{+}(\lambda)$, i.e., $s_{-}^{0}<u^{*}(x)<s_{+}^{0}$ and $0<u_{\max }^{*}<s_{+}^{0}$. We introduce a new parameter

$$
\eta=\eta(u)=\sigma\|u\|^{-2} .
$$

Then, for all $(\lambda, \sigma, u) \in \theta(\lambda)$ we have that $\eta \leqslant 0$ and we claim that $\eta$ covers the interval $(-\infty, 0]$ as $(\lambda, \sigma, u)$ ranges over $\theta(\lambda)$ : Clearly, if $\sigma \rightarrow 0$ and $(\lambda, \sigma, u) \rightarrow$ $\left(\lambda, 0, u^{*}\right)$ then $\eta \rightarrow 0$. On the other hand, if $\|u\| \rightarrow 0$, then we may consider problem (2.6)

$$
u=\lambda 巳 F(u)+\sigma w
$$

divide by $\|u\|$, let $\bar{v}=u\|u\|^{-1}$ and obtain

$$
\bar{v}=\lambda m_{0} \varrho \bar{v}+\lambda \mathscr{L}\left(o(\|u\|)\|u\|^{-1}\right)+\sigma\|u\|^{-1} w .
$$

Hence, $\sigma u^{-1} \rightarrow 0$, for otherwise the linear problem

$$
u=\lambda m_{0} \varrho u
$$

would have a nontrivial solution, which is impossible since $\lambda \neq \lambda_{i}^{0}, i=0,1,2, \ldots$ Hence, $\eta \rightarrow-\infty$ as $\|u\| \rightarrow 0, \sigma \rightarrow 0$ for $(\lambda, \sigma, u) \in \mathcal{O}(\lambda)$.

We next consider the equation

$$
v=\lambda \varrho\left(\|v\|^{2} F\left(v\|v\|^{-2}\right)\right)+\eta w, \quad \eta \in \mathbf{R},
$$

and observe that it shares essential properties with equation (2.6) with the nonlinear term $F$ replaced by the nonlinear term above (which no longer is the Nemits'kii operator associated with a function $f: \mathbf{R} \rightarrow \mathbf{R}$ ). The considerations of $\S 3$, nevertheless, may be applied, as one may check by verifying Lemmata 3.1, 3.2 and Corollary 3.1 appropriately stated for this equation. Analyzing solutions $(\lambda, \sigma, u) \in \vartheta(\lambda)$ (from the "inner world") is then equivalent to studying solutions of (4.23). In particular, if $(\lambda, \sigma, u) \rightarrow(\lambda, 0,0)$ then $\eta^{-1} v \rightarrow v^{\infty}$, where $v^{\infty}$ is the unique solution of (3.4). An analogue of Theorem 4.3 may hence be established to conclude that, in 
fact, $(\lambda, \eta, v)$ will lie on a subcontinuum $\tilde{\mathcal{L}}^{-}(\lambda) \subset\{\lambda\} \times(-\infty, 0] \times E$ of solutions of (4.26) with the same structural properties as $\mathcal{2}^{-}(\lambda)$. Note that $v$ satisfies the functional differential equation

$$
\begin{aligned}
L v+\lambda\|v\|^{2} f\left(v\|v\|^{-2}\right) & =0, & & x \in \Omega, \\
v & =\eta \phi, & & x \in \partial \Omega,
\end{aligned}
$$

and since $r_{+}^{\infty}=\left(s_{+}^{0}\right)^{-1}$ is the largest positive zero of the function $g(r)=r^{-2} f(r)$, it follows as in Theorem 4.3 that for $(\lambda, \eta, v) \in \mathcal{L}^{-}(\lambda), v_{\max }>\left(s_{+}^{0}\right)^{-1}$. Thus, the structure of the subcontinuum $\theta(\lambda)$ in Theorem 4.2 may be understood by studying the continuum $\tilde{\mathcal{L}}^{-}(\lambda)$ established by the related problem (4.27). These considerations will be exploited in greater detail in the next section.

REMARK 4.5. If we consider problem (2.3) with the boundary data $\phi \equiv 1$, then for every $\sigma$ such that $f(\sigma)=0, u \equiv \sigma$ will be a solution of (2.3). Hence, if $s_{i}$ is a zero of $f$, letting $v(x)=u(x)-s_{i}, \tilde{f}(\cdot)=f\left(\cdot+s_{i}\right)$ we obtain the problem

$$
\begin{gathered}
L v+\lambda \tilde{f}(v)=0, \quad x \in \Omega, \\
v=\sigma-s_{i}=\tilde{\sigma}, \quad x \in \partial \Omega,
\end{gathered}
$$

which is a problem similar to the one studied before. Hence, if $s_{i}$ is such that $f\left(s_{i}\right)=0$, then we may conclude via the previous results applied to (4.28) that the $\sigma$-continua of (2.3) will, in fact, contain $\left(\lambda, s_{i}, u \equiv s_{i}\right)$ and that the 'world' surrounding this solution will have a similar structure to the 'inner worlds' described earlier provided $f^{\prime}\left(s_{i}\right)>0$. This observation, in the context of continuation principles, has the obvious implication that any one of the stationary solutions may be chosen as a starting point for the $\sigma$-continuum. This may provide considerable computational savings in the computation of nontrivial solutions of the homogeneous problem whose norm is large.

5. Ordinary differential equations. The goal of this section is to establish Figures 1-4 for the ordinary differential equation

$$
\begin{aligned}
u^{\prime \prime}+\lambda f(u) & =0, \quad 0<x<\pi, \\
u(0) & =\sigma=u(\pi)
\end{aligned}
$$

which is a particular case of problem (2.3). Here $f$ is a $C^{1}$ function satisfying (2.2) and (2.8), e.g. Figure 12 gives a typical picture.

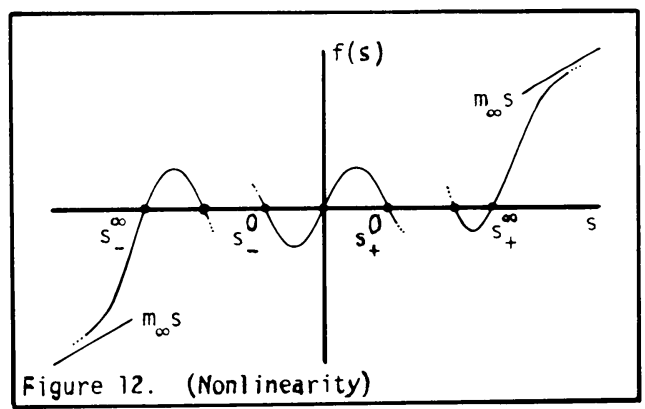


The essential features which we will exploit are the nodal structure of solutions together with the fundamental symmetries (2.14) and the properties of solutions of the linearization

$$
v^{\prime \prime}+\lambda m_{\infty} v=0, \quad v(0)=1=v(\pi) .
$$

We need the following properties of the explicit solutions $v_{k}^{\infty}(\lambda)$ (see (2.12)) of (5.2).

$$
\left\{\begin{array}{l}
\text { Let } \lambda m_{\infty} \neq n^{2}, n=1,2,3, \ldots \text { Then } v_{k}^{\infty}(\lambda) \text { is symmetric } \\
\text { about } \pi / 2 \text {. Furthermore, } v_{k}^{\infty}(\lambda) \text { and } v_{k+1}^{\infty}(\lambda), k=1,3,5, \ldots, \\
\text { have }(k+1) \text { interior zeros in }(0, \pi) \text { and these zeros are } \\
\text { simple. Moreover, }\left(v_{k}^{\infty}(\lambda)\right)^{\prime}(0) \cdot\left(v_{k}^{\infty}(\lambda)\right)^{\prime}(\pi)<0 \text { for all } \lambda m_{\infty} \\
\neq n^{2}, \text { and } \operatorname{sign}\left(v_{k}^{\infty}(\lambda)\right)^{\prime}(0)=(-1)^{k} ; \text { also } \\
\quad \operatorname{deg}\left(\text { id }-\lambda m_{\infty} \ell-1, B_{\varepsilon}\left(v_{k}^{\infty}(\lambda)\right), 0\right)=(-1)^{k}, \quad k=0,1,2, \ldots
\end{array}\right.
$$

Applying Theorem 3.2 to the homogeneous problem

$$
\begin{gathered}
u^{\prime \prime}+\lambda f(u)=0, \quad 0<x<\pi, \\
u(0)=0=u(\pi)
\end{gathered}
$$

we conclude that $\lambda_{k}^{\infty}=(k+1)^{2} m_{\infty}^{-1}, k=0,1,2, \ldots$, is a point of bifurcation from infinity, where continua $+\bigodot_{k}^{\infty},-\bigodot_{k}^{\infty} \subset \Lambda \times\{0\} \times E$ bifurcate which have the following properties:

$$
\left\{\begin{array}{l}
+e_{k}^{\infty},-e_{k}^{\infty} \text { are unbounded with respect to } \lambda ;(\lambda, 0, u) \in+e_{k}^{\infty}, \\
\text { then } u \text { has precisely } k \text { nodes in }(0, \pi) \text { and } u^{\prime}(0)>0 \\
(\lambda, 0, u) \in e_{k}^{\infty}, \text { then } u \text { has precisely } k \text { nodes in }(0, \pi) \text { and } \\
u^{\prime}(0)<0 .
\end{array}\right.
$$

A similar situation will be the case for bifurcation from zero at the bifurcation points $\lambda_{k}^{0}=(k+1)^{2} m_{0}^{-1}, k=0,1,2, \ldots$. The corresponding $\lambda$-continua will be denoted by $\pm e_{k}^{0}$.

For the discussion to follow we make the following assumption:

$$
\left\{\begin{array}{l} 
\pm \bigodot_{k}^{\infty} \text { and } \pm \bigodot_{k}^{0}, k=0,1,2, \ldots, \text { may be parametrized by } \lambda, \\
\text { and, there are no solutions }(\lambda, 0, u) \text { of }(5.4) \text { with }\left|s_{-}^{\infty}\right| \leqslant\|u\|< \\
\left|s_{-}^{0}\right| \text { or } s_{+}^{0} \leqslant\|u\|<s_{+}^{\infty} .
\end{array}\right.
$$

The first assumption, of course, is difficult to verify in practice and also includes the supposition that ${ }^{ \pm} \bigodot_{k}^{\infty}$ and ${ }^{ \pm} C_{k}^{0}$ do not undergo secondary bifurcation in $\Lambda \times\{0\} \times E$. Typical examples are discussed in [18]. The second assumption, e.g. would be guaranteed if $\int_{s_{+}^{0}}^{r_{+}} f(s) d s=0$ and $\int_{r_{-}}^{s_{-}^{0}} f(s) d s=0$ would imply that $r_{-}<s_{-}^{\infty}$ and $r_{+}>s_{+}^{\infty}$. Other conditions on $f$ are of course possible. Moroever, it follows from elementary degree calculations that if $(k=0,1,2, \ldots)$

$$
\begin{array}{ll}
(\lambda, 0, u) \in \pm \bigodot_{k}^{\infty}, & \text { then } \operatorname{deg}\left(\mathrm{id}-\lambda \mathcal{L} F, U_{\varepsilon}, 0\right)=(-1)^{k+1}, \\
(\lambda, 0, u) \in \pm \bigodot_{k}^{0}, & \text { then } \operatorname{deg}\left(\mathrm{id}-\lambda \mathcal{L} F, U_{\varepsilon}, 0\right)=(-1)^{k},
\end{array}
$$


where $U_{\varepsilon}$ is an isolating neighborhood of $u$ in $E$. Any solution of (5.4) is a particular segment of a periodic solution of the phase flow $\Phi^{x}, x \in \mathbf{R}$, corresponding to the vectorfield

$$
V(u, v)=(v,-\lambda f(u)) .
$$

Thus, any solution $(\lambda, 0, u)$ of $(5.4)$ for which $u^{\prime}(0) \cdot u^{\prime}(\pi)<0$ is symmetric about $\pi / 2$ as a consequence of (2.14). Hence, we may consider ${ }^{ \pm} \bigodot_{2 k}^{\infty}$ and $\pm \bigodot_{2 k}^{0}$ imbedded in the space

$$
E_{s}=\left\{u \in C^{0}[0, \pi]: u(\pi / 2-x)=u(\pi / 2+x)\right\}
$$

for $k=0,1,2, \ldots$. Solving (5.4) with respect to $E_{s}$ is equivalent to solving

$$
\begin{aligned}
u^{\prime \prime}+\lambda f(u) & =0, \quad 0<x<\pi / 2, \\
u(0) & =0=u^{\prime}(\pi / 2)
\end{aligned}
$$

in $E$. Problem (5.8), however, has only $\lambda_{2 k}^{0}$ and $\lambda_{2 k}^{\infty}, k=0,1,2, \ldots$, as bifurcation points from zero and infinity. It is important to note that the Leray-Schauder degree of $v_{k}^{\infty}(\lambda)$ computed with respect to $E$ or $E_{s} \subset E$ differs from that previously computed (see (5.3)),

$$
\operatorname{deg}_{E_{s}}\left(\text { id }-\lambda m_{\infty} E-1, B_{\varepsilon}\left(v_{k}^{\infty}(\lambda)\right), 0\right)=\left\{\begin{array}{cl}
+1, & k=0,3,4,7,8, \ldots, \\
-1, & k=1,2,5,6, \ldots,
\end{array}\right.
$$

where $B_{\varepsilon}(\cdot)$ is a neighborhood of $v_{k}^{\infty}(\lambda)$ in $E_{s}$. Likewise the degrees of solutions on $\pm \bigodot_{2 k}^{\infty}$ and ${ }^{ \pm} \bigodot_{2 k}^{0}$ change when considered in $E_{s}$ (compare with (5.7)):

$$
\begin{aligned}
& (\lambda, 0, u) \in \pm \bigodot_{2 k}^{\infty} \text { then } \operatorname{deg}_{E_{s}}\left(\text { id }-\lambda £ F, U_{\varepsilon}, 0\right)=(-1)^{k+1}, \\
& (\lambda, 0, u) \in \pm \bigodot_{2 k}^{0} \text { then } \operatorname{deg}_{E_{s}}\left(\text { id }-\lambda £ F, U_{\varepsilon}, 0\right)=(-1)^{k},
\end{aligned}
$$

where $U_{\varepsilon}$ is an isolating neighborhood of $u$ in $E_{s}$. The signs attached to solutions in Figures 1-4 are to be read as follows: signs without brackets are degrees in $E$, whereas signs in brackets are degrees in $E_{s}$. Furthermore, we note that

$$
(\mathrm{id}-\lambda E-\sigma \mathrm{l})\left(E_{s}\right) \subset E_{s}
$$

and, therefore, we may apply the theory developed in $\$ \S 3$ and 4 in the subspace $E_{s}$. We are now able to establish Figures 1 and 2. For simplicity, we assume furthermore that $m_{0}=m_{\infty}$, so that $\lambda_{k}^{0}=\lambda_{k}^{\infty}, k=0,1,2, \ldots$ If $\lambda<\lambda_{0}^{0}=\lambda_{0}^{\infty}$, then (5.4) has no nontrivial solution and the picture for $\delta(\lambda)$ (according to Theorem 3.1) in Figure 1 follows from Theorem 3.1.

To derive Figure 2, let $\lambda_{0}^{0}=\lambda_{0}^{\infty}<\lambda<\lambda_{1}^{0}=\lambda_{1}^{\infty}$. Observe that $v_{1}^{\infty}(\lambda)$ has a negative minimum and, therefore, for any

$$
(\lambda, \sigma, u) \in \mathscr{2}^{+}(\lambda), u \text { has a negative minimum below } s_{-}^{\infty}
$$

and, for any

$$
(\lambda, \sigma, u) \in \mathcal{2}^{-}(\lambda), u \text { has a positive maximum above } s_{+}^{\infty} .
$$

This establishes the $\sigma$-subcontinua $\mathcal{Q}^{+}(\lambda)$ and $\mathcal{L}^{-}(\lambda)$ in Figure 2. Applying Theorems 4.1 and 4.2 we obtain the $\sigma$-subcontinua $\mathscr{N}(\lambda), \mathscr{P}(\lambda)$ and $\mathcal{O}(\lambda)$. This establishes Figure 2. 
To derive Figure 3 let $\lambda_{1}^{0}=\lambda_{1}^{\infty}<\lambda<\lambda_{2}^{0}=\lambda_{2}^{\infty}$, and analyze the results from $\S \S 3$ and 4 in more detail. While all above results are also true for the general problem (2.3), the following is very special for (5.4). Firstly, we notice a fundamental obstruction: we may look at the $\sigma$-subcontinua $2^{+}(\lambda)$ and $2^{-}(\lambda)$ (according to Theorem 4.3) both in $E$ and $E_{s}$. In $E_{s}$ problem (5.4) does not have solutions $(\lambda, 0, u)$ with $u^{\prime}(0) \cdot u^{\prime}(\pi)>0$ (i.e. $u=u_{1}^{\infty}$ in Figure 3). In fact, for $\lambda$ as chosen above, problem (5.4) has in $E_{s}$ only solutions $(\lambda, 0, u)$ with

$$
u(x)>0 \text { or } u(x)<0 .
$$

Thus, Theorem 4.3 yields $\sigma$-subcontinua $\mathcal{2}_{s}^{+}(\lambda)$ and $\mathcal{2}_{s}^{-}(\lambda)$ in $\{\lambda\} \times[0, \infty) \times E_{s}$ and $\{\lambda\} \times(-\infty, 0] \times E_{s}$, respectively, connecting a solution of (5.1) near $\left(\lambda, \sigma, \sigma v_{2}^{\infty}(\lambda)\right)$ with a negative solution of $(5.4)$ in $-e_{0}^{\infty}(\lambda)$ and a solution of $(5.1)$ near $\sigma v_{2}^{\infty}(\lambda)$ with a positive solution in ${ }^{+} e_{0}^{\infty}(\lambda)$, respectively. If, however, we consider these $\sigma$-subcontinua $\mathscr{2}_{s}^{+}(\lambda)$ and $\mathscr{2}_{s}^{-}(\lambda)$ in the total space $E$ we observe an obstruction due to the homotopy property of degree and the calculations (5.3), (5.7), (5.9) and (5.10). In other words, $\mathscr{2}^{+}(\lambda)$ cannot be identical with $\mathscr{2}_{s}^{+}(\lambda)$, and $2^{-}(\lambda)$ cannot be identical with $\mathcal{2}_{s}^{-}(\lambda)$. And we conclude that along $\mathscr{2}_{s}^{+}(\lambda)$ and $\mathcal{2}_{s}^{-}(\lambda)$ in $\{\lambda\} \times \Sigma \times E$ there must be a secondary bifurcation, because, e.g.

$$
\operatorname{deg}\left(\mathrm{id}-\lambda \varrho F-\sigma w, \Gamma_{\sigma}(\varepsilon), 0\right)=\left\{\begin{array}{cc}
+1, & \sigma \gg 1, \\
-1, & \sigma=0,
\end{array}\right.
$$

where $\Gamma(\varepsilon)$ is a tubular neighborhood of $\mathcal{Q}_{s}^{+}(\lambda)$ in $\{\lambda\} \times[0, \infty) \times E$ and $\Gamma_{\sigma}(\varepsilon)$ is its $\sigma$-section. A similar obstruction occurs, if one considers $\theta_{s}(\lambda)$ according to Theorem 4.2 in $\{\lambda\} \times \Sigma \times E_{s}$ and $\vartheta(\lambda)$ according to Theorem 4.2 in $\{\lambda\} \times \Sigma \times E$ (see Figure 3). Again a secondary bifurcation along $\Theta_{s}(\lambda)$ must occur. These secondary bifurcations are typical for all parameter intervals

$$
\lambda_{2 k-1}^{0}=\lambda_{2 k-1}^{\infty}<\lambda<\lambda_{2 k}^{0}=\lambda_{2 k}^{\infty}, \quad k=1,2,3, \ldots,
$$

because there the relevant degrees along $\mathcal{2}_{s}^{+}(\lambda), \mathcal{2}_{s}^{-}(\lambda)$ and $\mathcal{O}_{s}(\lambda)$ provide an obstruction according to (5.3), (5.7), (5.9) and (5.10). These bifurcations go along with another very singular phenomenon and that is the existence of $\sigma$-subcontinua in $\{\lambda\} \times \Sigma \times E$ which are homeomorphic to $S^{1}$.

LEMMA 5.1 ( $\sigma$-CONTINUA). Let $\lambda$ satisfy (5.11). Then under the above assumptions on $f$, problem (5.1) admits $\sigma$-subcontinua

$$
\varsigma_{2 k-1}^{\infty}(\lambda), \varsigma_{2 k-1}^{0}(\lambda) \subset\{\lambda\} \times \Sigma \times E \backslash\{0\}
$$

such that

$$
\varsigma_{2 k-1}^{\infty}(\lambda) \cong S^{1} \cong \varsigma_{2 k-1}^{0}(\lambda),
$$

and these bifurcate from infinity at $\lambda_{2 k-1}^{\infty}$ (resp. from zero at $\lambda_{2 k-1}^{0}$ ) in $\Lambda \times \Sigma \times E$.

Proof. If $\lambda$ satisfies (5.11), then the homogeneous problem (5.4) admits the following solutions: $\left(\lambda, 0,{ }^{ \pm} u_{2 k-1}^{\infty}\right) \in{ }^{ \pm} \bigodot_{2 k-1}^{\infty}(\lambda),\left(\lambda, 0,{ }^{ \pm} u_{2 k-1}^{0}\right) \in{ }^{ \pm} \bigodot_{2 k-1}^{0}(\lambda)$ such that

$$
\begin{array}{cc}
\left({ }^{+} u_{2 k-1}^{\infty}\right)_{\max }>s_{+}^{\infty}, & \left({ }^{-} u_{2 k-1}^{\infty}\right)_{\min }<s_{-}^{\infty}, \\
0<\left({ }^{+} u_{2 k-1}^{0}\right)_{\max }<s_{+}^{0}, & 0>\left({ }^{-} u_{2 k-1}^{0}\right)_{\min }>s_{-}^{0}
\end{array}
$$


and all four solutions have precisely $(2 k-1)$ simple zeros in $(0, \pi)$. Now let $u$ be any of these four solutions. Then $\left(u(x), u^{\prime}(x)\right), x \in[0, \pi]$, constitutes a smooth periodic orbit for the phase flow $\Phi^{x}$ corresponding to the vectorfield $V(u, v)=$ $(v,-\lambda f(u))$ enclosing the origin (use (2.14)). Thus, the desired $\sigma$-continua are obtained by a simple shift along the trajectory given by $u$.

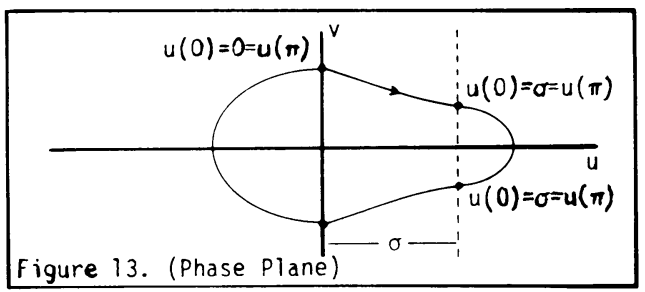

THEOREM 5.1 ( $\sigma$-CONTINUA). Let $\lambda$ satisfy (5.11). Then under the above assumptions on $f$, problem (5.1) admits secondary bifurcations which are given by

$$
{\mathcal{Q}_{s}^{+}}^{+}(\lambda) \cap \mathfrak{S}_{2 k-1}^{\infty}(\lambda) \text { and } \mathscr{Q}_{s}^{-}(\lambda) \cap \varsigma_{2 k-1}^{\infty}(\lambda), \quad \hat{O}_{s}(\lambda) \cap \varsigma_{2 k-1}^{0}(\lambda) .
$$

Proof (cf. Figure 14). $\mathcal{Q}_{s}^{+}(\lambda), \mathcal{L}_{s}^{-}(\lambda)$ and $\Theta_{s}(\lambda)$ are obtained in $\{\lambda\} \times \Sigma \times E_{s}$ as a consequence of Theorems 4.2 and 4.3. Since $\left(v_{2 k}^{\infty}(\lambda)\right)^{\prime}(0)>0$ and $\left(v_{2 k}^{\infty}(\lambda)\right)^{\prime}(\pi)<0$ and $v_{2 k}^{\infty}(\lambda)$ has $2 k$ simple zeros in $(0, \pi)$, we may conclude from Lemma 4.4 that $\mathcal{Q}_{s}^{+}(\lambda)$ connects to a solution $(\lambda, 0, u)$ of (5.4) such that $u$ has $k$ negative minima below $s_{-}^{\infty}$ and $u^{\prime}(0)<0, u^{\prime}(\pi)>0$. Therefore, there must be a $\sigma=\sigma_{b}>0$ and $\left(\lambda, \sigma_{b}, u_{b}\right) \in \mathscr{Q}_{s}^{+}(\lambda)$ such that $u_{b}^{\prime}(0)=0=u_{b}^{\prime}(\pi)$. This may be seen because solutions in $\mathcal{Q}_{s}^{+}(\lambda)$ vary continuously even in the $C^{1}$ topology. As in the proof of Lemma 5.1, $u_{b}$ constitutes a periodic orbit for the phase flow $\Phi^{x}$ and therefore gives rise to another $\sigma$-continuum which is homeomorphic to $S^{1}$ and which intersects $\{\lambda\} \times\{0\} \times$ $E$. Thus, due to (5.6), $\left(\lambda, \sigma_{b}, u_{b}\right)$ is on $\varsigma_{2 k-1}^{\infty}(\lambda)$ and $\sigma_{b}=\left(u_{b}\right)_{\max }=\left({ }^{+} u_{2 k-1}^{\infty}\right)_{\max }$. The arguments for $\mathcal{L}^{-}(\lambda)$ and $\mathcal{\Theta}_{s}(\lambda)$ are similar.

Results in [18] may be used to prove that the above established secondary bifurcations along $\varsigma_{2 k-1}^{\infty}(\lambda)$ and $\varsigma_{2 k-1}^{0}(\lambda)$ are the only possible ones.

Putting our earlier considerations and Lemma 5.1 as well as Theorem 5.1 together we have established Figure 3.

To derive Figure 4 let $\lambda_{2}^{0}=\lambda_{2}^{\infty}<\lambda<\lambda_{3}^{0}=\lambda_{3}^{\infty}$. Our first observation is that while $v_{1}^{\infty}(\lambda)$ and $v_{2}^{\infty}(\lambda)$ have the same nodal structure, $v_{3}^{\infty}(\lambda)$ has a new different nodal structure, i.e. $v_{3}^{\infty}(\lambda)$ has four interior simple zeros in $(0, \pi)$ and two negative minima and one positive maximum. Therefore, $\mathcal{2}^{+}(\lambda)$ (resp. $\mathcal{2}^{-}(\lambda)$ ) will connect a solution near $\sigma v_{3}^{\infty}(\lambda), \sigma \gg 1$ (resp. $\left.\sigma \ll-1\right)$, with a solution in ${ }^{+} \bigodot_{2}^{\infty}(\lambda)\left(\right.$ resp. $\left.{ }^{-} e_{2}^{\infty}(\lambda)\right)$. The $\sigma$-subcontinua $\delta_{1}^{\infty}(\lambda)$ and $\varsigma_{1}^{0}(\lambda)$ homeomorphic to $S^{1}$ will still exist together with their secondary bifurcations. The bifurcating solutions $\left(\lambda, \sigma_{b}, u_{b}\right)$ (see the proof of Theorem 5.1) will also maintain the connections established in Figure 3 except, that e.g. the solution $\left(\lambda, \sigma_{b}, u_{b}\right)$ displayed in Figure 14 can now not be continued to a 


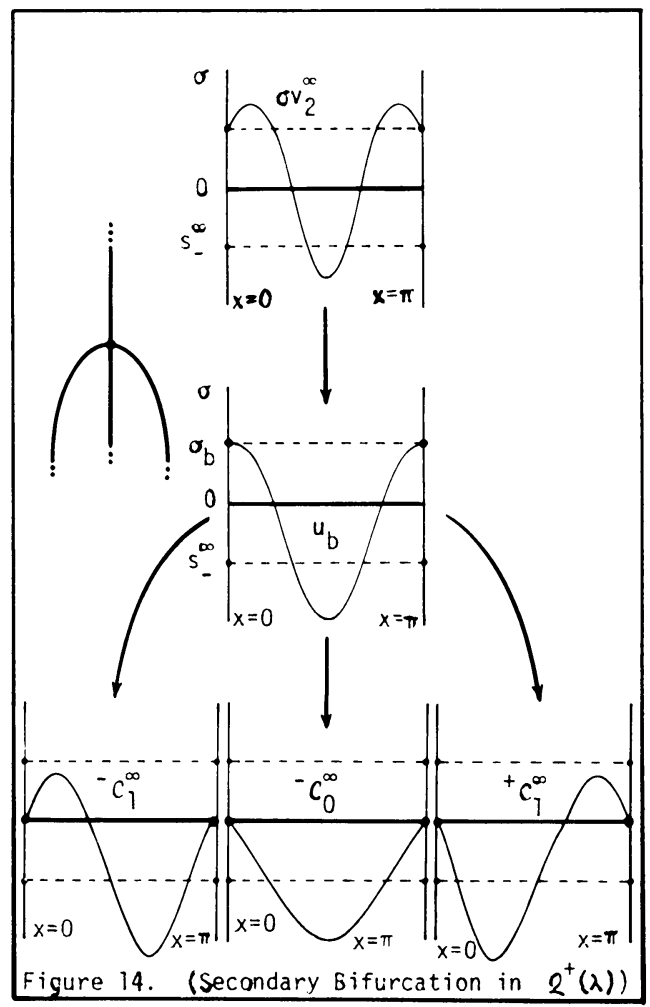

solution near $\left(\lambda, \sigma, \sigma v_{3}\right)$ in $\{\lambda\} \times[0, \infty) \times E_{s}$ because $u_{b}$ and $v_{3}^{\infty}$ differ in the number of negative minima. For topological reasons

$$
\left(\operatorname{deg}_{E_{s}}\left(\mathrm{id}-\lambda \varrho F-\sigma_{b} 1, B_{\varepsilon}\left(u_{b}\right), 0\right)=-1\right),
$$

however, $\left(\lambda, \sigma_{b}, u_{b}\right)$ must be continuable in $\{\lambda\} \times[0, \infty) \times E_{s}$. Since all solutions of (5.1) which do not have precisely two negative minima are 'a priori' bounded in $\{\lambda\} \times[0, \infty) \times E$ (see Lemmata 3.1 and 3.2 ), we may conclude by continuation arguments similar to those used in $\S \S 3$ and 4 , that $\left(\lambda, \sigma_{b}, u_{b}\right)$ must be continuable in $\{\lambda\} \times[0, \infty) \times E_{s}$ to a solution in $\{\lambda\} \times\{0\} \times E_{s}$. Since the negative minimum must be preserved and since no further negative minima can be introduced along this continuum, we may conclude that $\left(\lambda, \sigma_{b}, u_{b}\right)$ is continuable to a solution in ${ }^{+} e_{2}^{\infty}(\lambda)$. Similar arguments can be used for the second bifurcating solution on $\delta_{1}^{\infty}(\lambda)$. Finally, we may exploit the considerations at the end of $\S 4$ together with the previous results to establish the configuration near $(\lambda, 0,0)$. This establishes Figure 4.

REMARK 5.1. (1) Our discussion above shows that as $\lambda$ increases, the schematics become more and more involved, but it is clear how the continuum diagram evolves as $\lambda$ crosses $\lambda_{k}^{0}=\lambda_{k}^{\infty}$; e.g. as $\lambda$ increases through $\lambda_{k}^{0}=\lambda_{k}^{\infty}, k$ odd, a pair of new continua $\delta_{k}^{\infty}(\lambda) \cong S^{1} \cong \delta_{k}^{0}(\lambda)$ are added, which are connected to the previously 
established configuration via two pairs of secondary bifurcations (see transition from Figure 2 to 3 ).

(2) So far, we have assumed that $\lambda_{k}^{0}=\lambda_{k}^{\infty}$. This assumption need not be made, since we only need to establish the 'outer world' and the 'inner world' separately and then use Theorem 4.1 to establish a connection between the two 'worlds'. Indeed, there is an obvious order principle in the global $\sigma$-continuum $\delta(\lambda)$ established in Theorem 3.1: beginning with a solution near $\left(\lambda, \sigma, \sigma v_{k}^{\infty}(\lambda)\right), \sigma \gg 1$, s first connects to a solution of (5.4) of large norm with the highest possible nodal structures prescribed by $v_{k}^{\infty}(\lambda)$. Then in descending order (with respect to the number of interior zeros), it connects to all other possible solutions of large norm until it arrives at the negative solution of large norm. A similar structure holds, if one follows along $\delta(\lambda)$ for $\sigma \ll-1$ to finally arrive at the positive solution of large norm. This constitutes the 'outer world'. Now there are connections to the 'inner world', which are independent from the relative relations of $\lambda_{k}^{\infty}$ and $\lambda_{k}^{0}$. These are provided by the continua $\mathscr{P}(\lambda)$ and $\mathcal{X}(\lambda)$, which are in some sense universal for problem (5.1). They connect the negative (positive) solution of large norm to the negative (positive) solution of small norm. From there a process analogous to the one in the outer world' continues. The negative (positive) solution of small norm is connected in ascending order (with respect to the number of interior zeros) to all other possible solutions of small norm until the solutions with the highest nodal structure are included. These are then connected to $(\lambda, 0,0)$.

(3) In particular the continua $\varsigma_{2 k-1}^{\infty}$ and $\varsigma_{2 k-1}^{0}$ are very intimately connected with the fundamental symmetries of the phase flow $\Phi^{x}$, characteristic of problem (5.1). If, e.g., one would choose the boundary conditions

$$
u(0)=\sigma, \quad u(\pi)=-\sigma,
$$

one could use similar symmetry considerations to obtain a complete description of $\delta(\lambda)$.

(4) Also, phase plane arguments easily convince one that the continua homeomorphic to $S^{1}$ will be destroyed if one of the boundary conditions is changed slightly, e.g.

$$
u(0)=\sigma, \quad u(\pi)=(1-\varepsilon) \sigma
$$

for $0<\varepsilon \ll 1$.

(5) If we apply Remark 4.5 to the ordinary differential equations just studied we see that (for $\sigma$ sufficiently large) there must be $S^{1} \sigma$-subcontinua surrounding those constant solutions which correspond to elliptic stationary points of the phase flow, and hence the 'inner world' described around the zero solution will be reproduced around elliptic stationary points; the particular structure of such a world, of course, depends upon $f^{\prime}\left(s_{i}\right)$, where $s_{i}$ is such a stationary point. This explains the existence of the two additional $S^{1}$ subcontinua in Figure 22, which is for the nonlinearity whose phase portrait is given by Figure 16. 

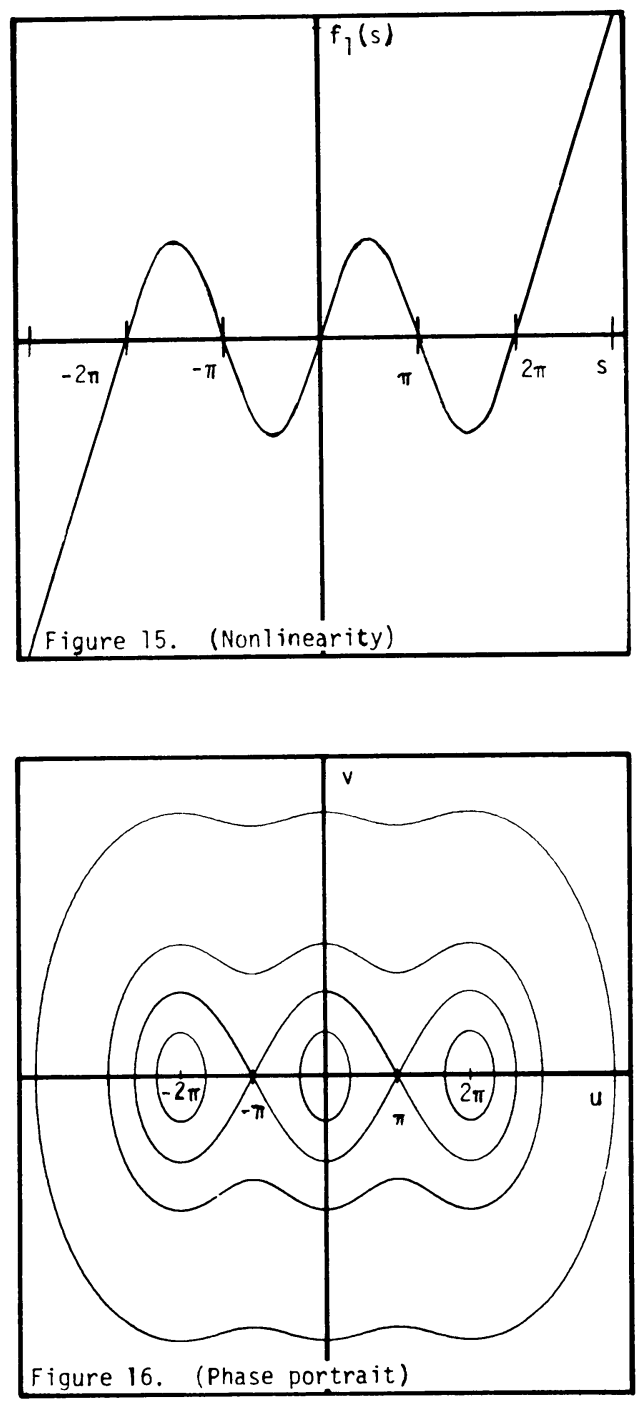

6. Numerical experiments. In this section we want to present some numerical experiments illustrating our theoretical investigations. These experiments were carried out by D. Saupe (University of Bremen) on the basis of the software package SCOUT. SCOUT (= Simplicial COntinuation UTilities) is an interactive package which is designed for the numerical study of nonlinear eigenvalue and bifurcation problems. It was developed at the University of Bremen in 1981/82. For a background of these methods see [8].

Our experiments are based on the following four nonlinearities:

$$
f_{1}(s)= \begin{cases}\sin (s), & -2 \pi \leqslant s \leqslant 2 \pi, \\ s-2 \pi, & s \geqslant 2 \pi \\ s+2 \pi, & s \leqslant-2 \pi\end{cases}
$$




$$
\begin{aligned}
& f_{2}(s)=s-s^{3}, \\
& f_{3}(s)=\left(s-s^{3}\right) \exp (a s), \\
& f_{4}(s)=\left(s-s^{3}\right)\left(1+a s^{2}\right) .
\end{aligned}
$$

Figure 15 shows the graph of $f_{1}$ and Figure 16 displays the phase portrait of the phase flow corresponding to

$$
u^{\prime}=v, \quad v^{\prime}=-\lambda f_{1}(u) .
$$

In particular, we see that the rest points $(-2 \pi, 0),(0,0)$ and $(2 \pi, 0)$ are elliptic and give rise to small periodic solutions in their neighborhood. Due to the symmetries of the phase flow $\Phi^{x}$ these give rise to $\sigma$-continua homeomorphic to $S^{1}$ of (5.1) provided (see Figure 22) $\lambda>\lambda_{1}^{0}=4$. Figures $17-20$ are a series of the $\sigma$-continua $\mathcal{Q}(\lambda)$ of (5.1) based on $f_{1}$ for the following choices of $\lambda$ :
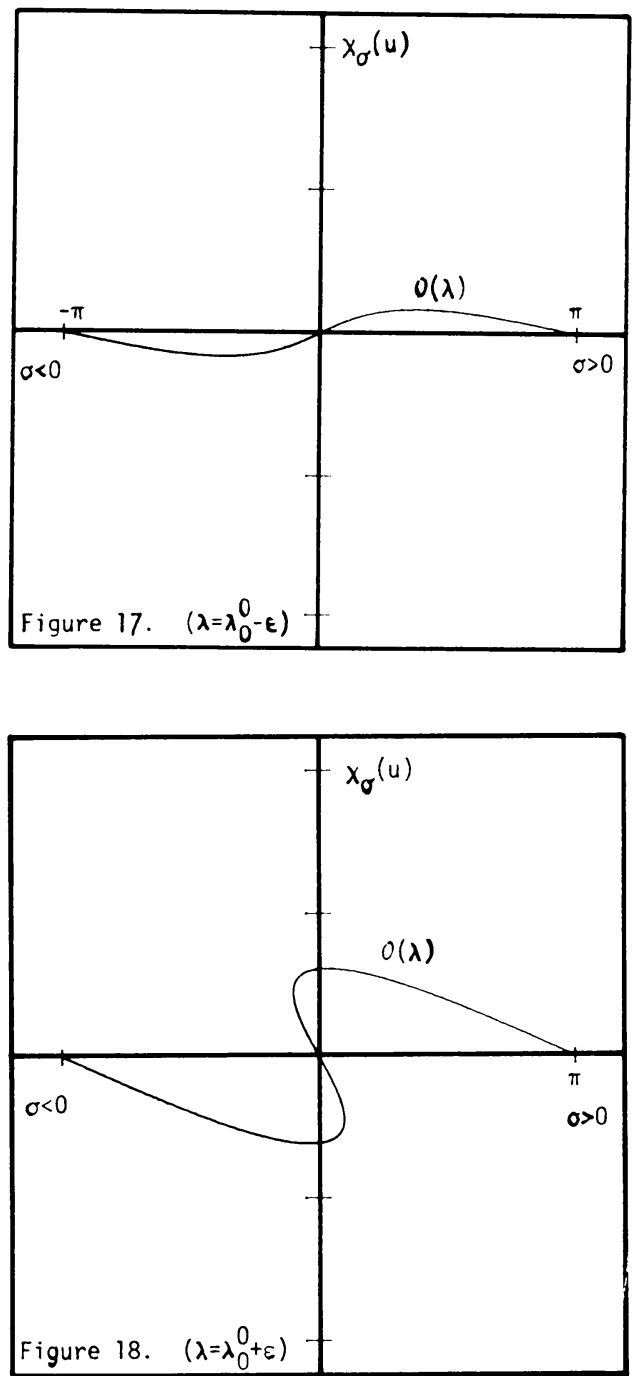

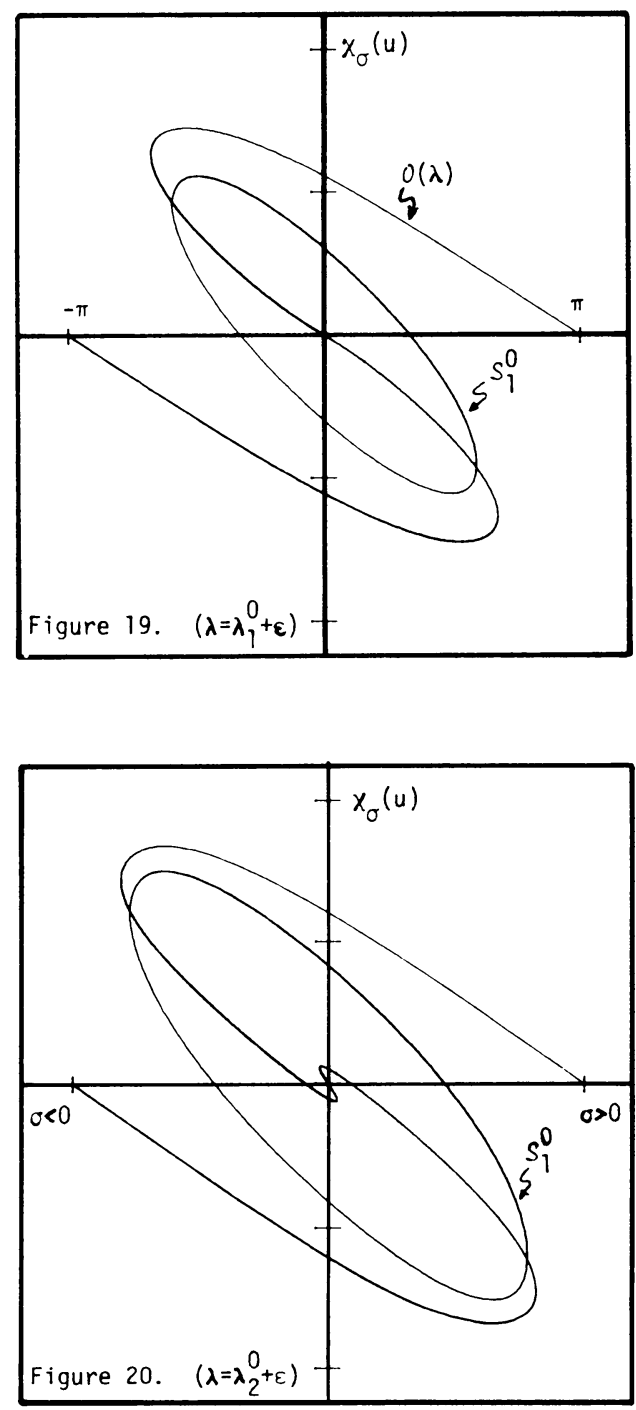

These figures as well as Figures 21-24 show $\sigma$-continua for fixed $\lambda$ in $\left(\sigma, \chi_{\sigma}\right)$ coordinates, where $\chi_{\sigma}$ is the functional $((\lambda, \sigma, u) \in \Lambda \times \Sigma \times E)$

$$
\chi_{\sigma}(u)=\int_{0}^{\pi / 2}(u(s)-\sigma) d s
$$

In particular, Figures $17-20$ indicate how the 'inner world' evolves as $\lambda$ is changed.

Comparing Figure 17 with Figure 1 there is danger of confusion: These two figures should represent the same continua qualitatively; they are, however, mirror images of each other. We have chosen Figure 1 in that particular form only to be consistent with Figures $2-4$. One may think of Figure 1 as representing e.g. $-\chi_{\sigma}$. 
Figures 21 and 22 give a sketch of the global $\sigma$-continuum $\delta$ (see Theorem 3.1) of (5.1) based on $f_{1}$ for the following choices of $\lambda$ :
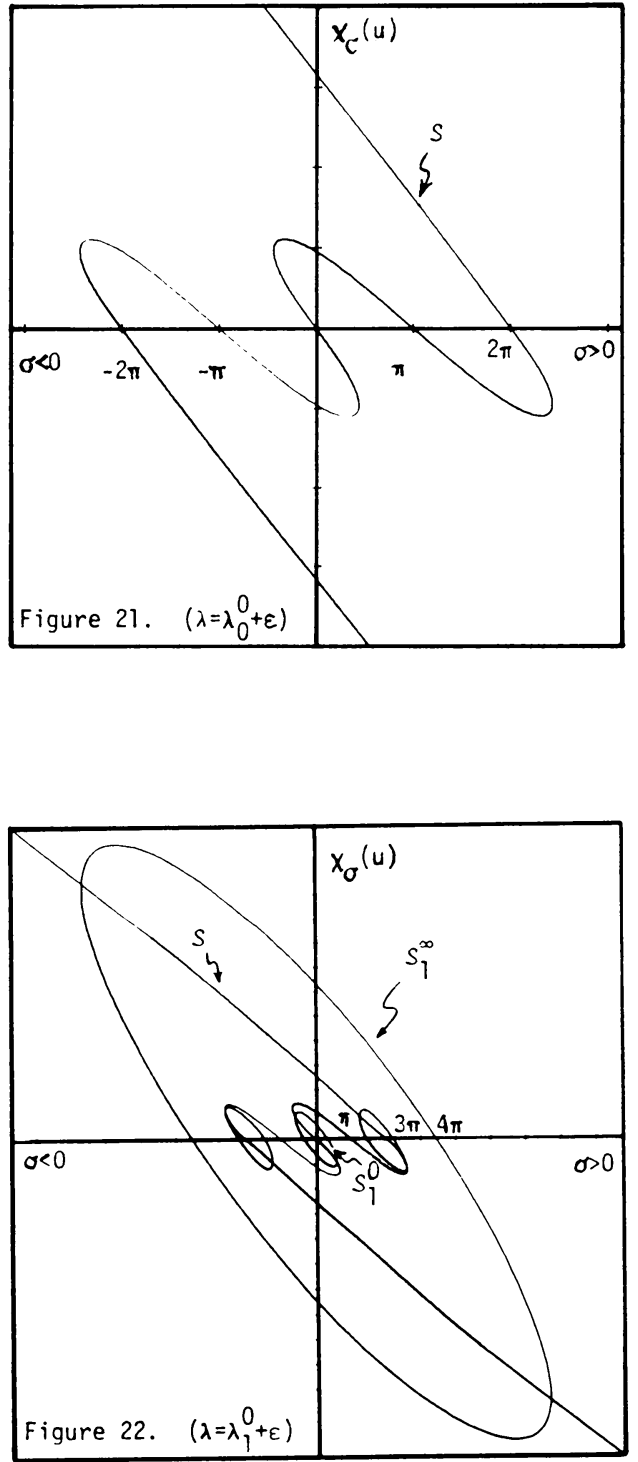


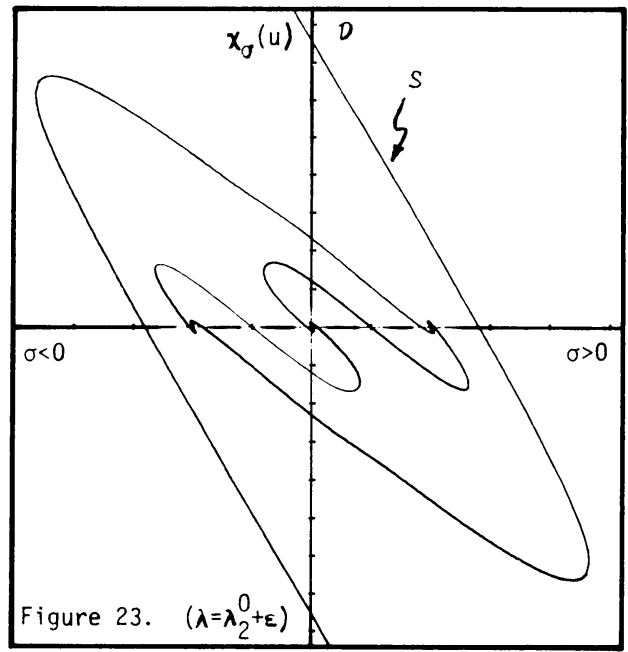

In particular we see the continua $\S_{1}^{0}$ and $\S_{1}^{\infty}$ as well as continua homeomorphic to $S^{1}$ corresponding to the rest points $(-2 \pi, 0)$ and $(2 \pi, 0)$ of $\Phi^{x}$ as they bifurcate from $\delta$. In order to better understand the structure of these continua we have omitted in Figure 23 the continua homeomorphic to $S^{1}$.

Finally, Figures 24-28 are a series of plots of two-dimensional continua of solutions of (5.1). These two-dimensional continua were numerically established by computing successive $\lambda$ - and $\sigma$-sections. Figures 24-28 show $\left(\lambda, \sigma, \chi_{\sigma}\right)$-coordinates of such solutions. In Figures $24-26$ we have $f=f_{2}$, i.e. the homogeneous problem

$$
u^{\prime \prime}+\lambda f(u)=0, \quad u(0)=0=u(\pi)
$$

has a supercritical bifurcation of positive solutions at $\lambda_{0}^{0}=1$ and of solutions with one internal zero at $\lambda_{1}^{0}=4$. Figure 24 shows the unfolding of the bifurcations at $\lambda_{0}^{0}$ in the two-parameter problem (5.1) (see Figures 8-10). Figures 25-26 show $(\lambda, \sigma)$ continua near $\left(\lambda_{0}^{0}, \sigma, 0\right)$ and $\left(\lambda_{1}^{0}, \sigma, 0\right)$ in two projections. Figure 26 is the projection of the two-dimensional continuum shown in Figure 25 onto the plane $\sigma \equiv 0$.

In Figure 27 we have $f=f_{3}$, i.e. the homogeneous problem (6.6) has a transcritical bifurcation of positive solutions at $\lambda_{0}^{0}=1$. Figure 27 shows this $\lambda$-bifurcation embedded into the two-dimensional continuum of solutions of (5.1).

In Figure 28 we have $f=f_{4}$, i.e., the homogeneous problem (6.6) has a subcritical bifurcation of positive solutions at $\lambda_{0}^{0}=1$. Figure 28 shows this $\lambda$-bifurcation embedded into the two-dimensional continuum of solutions of (5.1).

In particular, Figures 24-28 indicate that the two-parameter problem (5.1) unfolds the $\lambda$-bifurcation at $\lambda_{0}^{0}$ regardless of the local nature of $f$ near zero. 


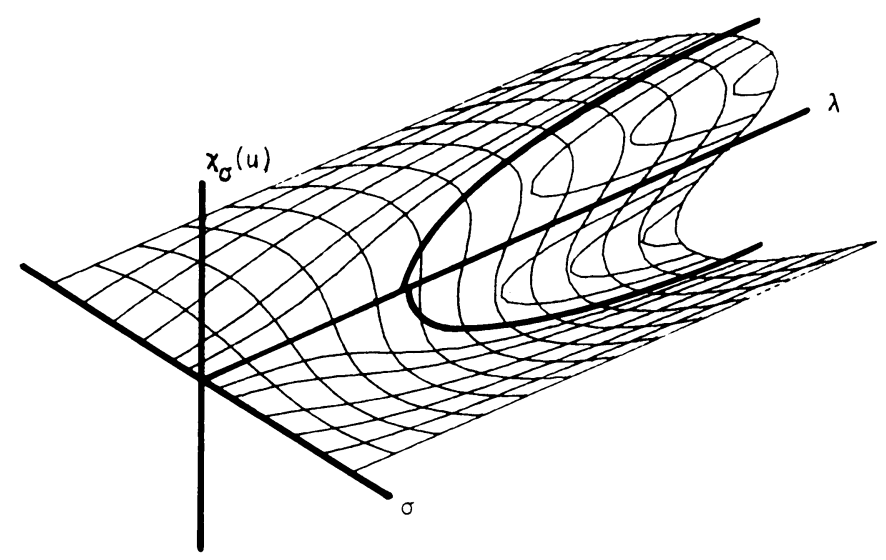

Figure 24. (Supercritical case, $f=f_{2}, 0 \leqslant \lambda<\lambda_{1}^{0}$ )

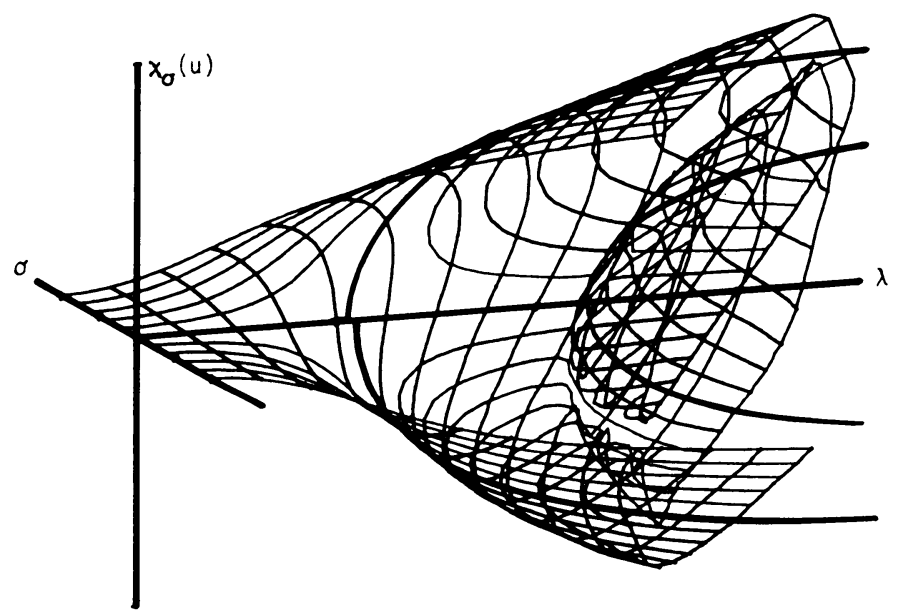

Figure 25. $\left(f=f_{2}, 0<\lambda<\lambda_{2}^{0}\right)$ 

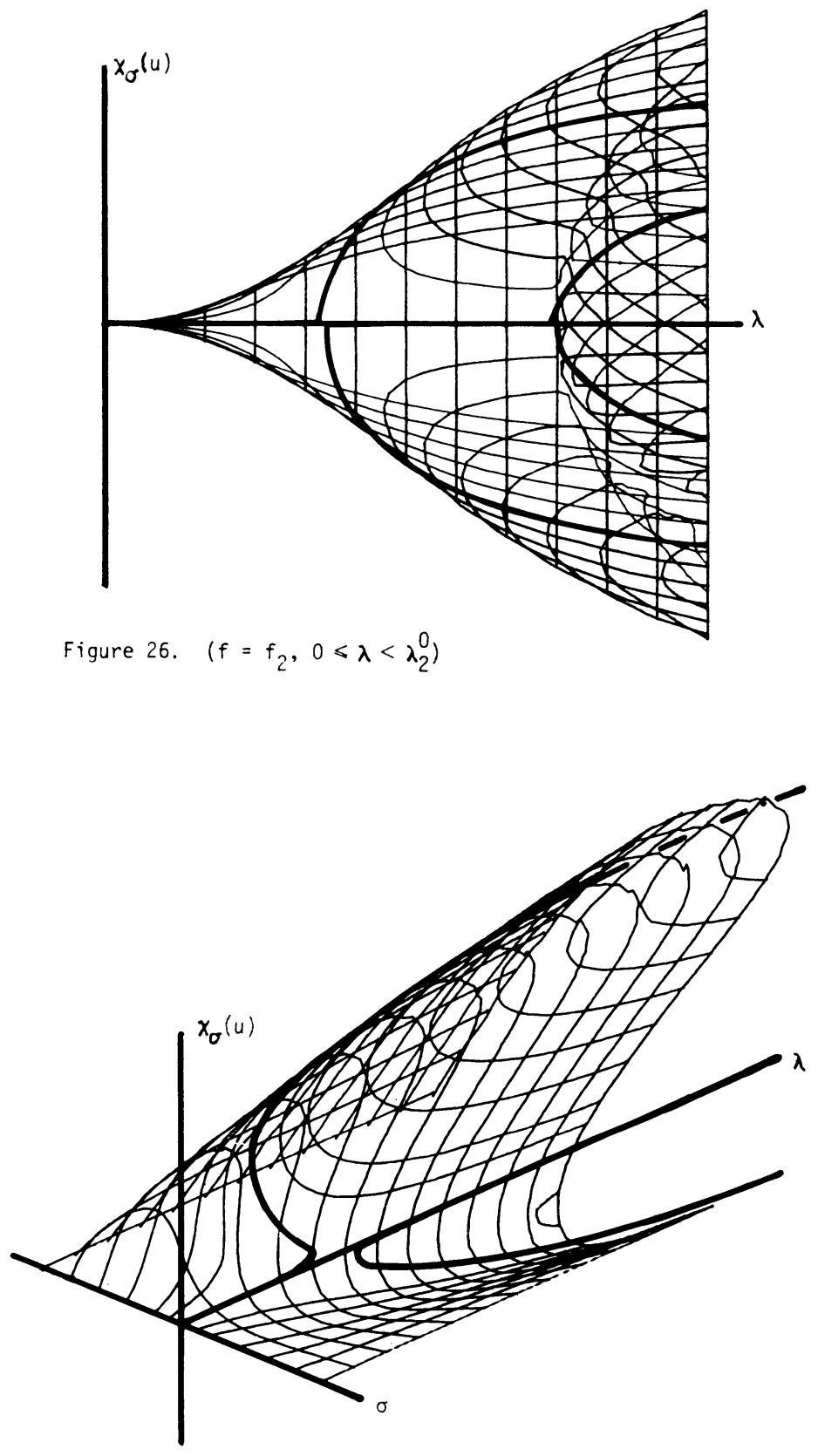

Figure 27. (transcritical case, $f=f_{3}, 0<\lambda<\lambda_{1}^{0}$ ) 


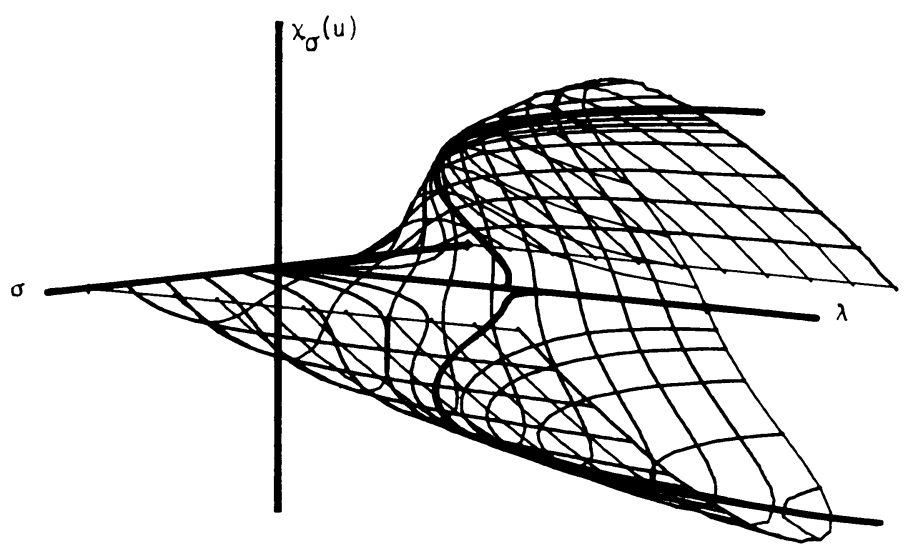

Figure 28. (Subcritical case, $f=f_{3}, 0<\lambda<\lambda_{1}^{0}$ )

\section{REFERENCES}

1. J. C. Alexander, A primer on connectivity, Lecture Notes in Math., vol. 886, Springer-Verlag, Berlin and New York, 1981, pp. 455-483.

2. A. Ambrosetti and P. Hess, Positive solutions of asymptotically linear elliptic eigenvalue problems, J. Math. Anal. Appl. 73 (1980), 411-422.

3. K. J. Brown and H. Budin, On the existence of positive solutions for a class of semilinear elliptic boundary value problems, SIAM J. Math. Anal. 10 (1979), 875-883.

4. D. Gilbarg and S. Trudinger, Elliptic partial differential equations of second order. Springer-Verlag. Berlin and New York, 1977.

5. P. Hess, On multiple positive solutions of nonlinear elliptic eigenvalue problems. Comm. Partial Differential Equations 6 (1981), 951-961.

6. K. Kuratowski, Topologv, PWN, Warsaw, 1950.

7. J. Leray and J. Schauder, Topologie et equations fonctionelles, Ann. Sci. Ecole Norm. Sup. 51 (1934). 45-78.

8. H. O. Peitgen, Topologische Perturbationen beim globalen numerischen Studium nichtlinearer Eigenwert-und Verzweigungsprobleme, Jahresber. Deutsch. Math.-Verein. 84 (1982), 107-162.

9. H. O. Peitgen and K. Schmitt, Perturbations topologiques globales des problèmes non linéaires aux valeurs propres, C. R. Acad. Sci. Paris Sér. A 291 (1980), 271-274.

10. Positive and spurious solutions of nonlinear eigenvalue problems, Lecture Notes in Math., vol. 878, Springer-Verlag, Berlin and New York, 1981, pp. 275-324.

11. Global topological perturbations of nonlinear elliptic eigenvalue problems, Math. Methods Appl. Sci. 5 (1983).

12. H. O. Peitgen, D. Saupe and K. Schmitt, Nonlinear elliptic boundary value problems versus their finite difference approximations: numerically irrelevant solutions, J. Reine Angew. Math. 322 (1981), 74-117.

13. P. H. Rabinowitz, Some aspects of nonlinear eigenvalue problems, Rocky Mountain J. Math. 3 (1973), 162-202.

14. On bifurcation from infinity, J. Differential Equations 14 (1973), 462-475.

15. K. Schmitt, Boundary value problems for quasilinear second order elliptic equations. Nonlinear Anal. 2 (1978), 263-309.

16.

- A study of eigenvalue and bifurcation problems for nonlinear elliptic partial differential equations via topo!ogical continuation methods, Notes de Séminaire de Mathématique, CABAY, Louvainla-Neuve, Belgium, 1982.

17. K. Schmitt and H. Smith, On eigenvalue problems for nondifferentiable mappings. J. Differential Equations 33 (1979), 294-319. 
18. J. Smoller, A Tromba and A. Wasserman, Nondegenerate solutions of boundary value problems, Nonlinear Anal. 4 (1980), 207-215.

19. C. A. Stuart, Concave solutions of singular nonlinear differential equations, Math. Z. 136 (1974), 117-135

20. C. A. Swanson, Comparison and oscillation theory of linear differential equations, Academic Press, New York, 1968.

21. G. T. Whyburn, Topological analysis, Princeton Univ. Press, Princeton, N.J., 1958.

ForschungsschWerpunkt Dynamische Systeme, Universität Bremen, D2800 Bremen, WeSt Germany

Department of Mathematics, University of Utah, Salt lake City, Utah 84112 